\title{
LOW-ENERGY NEUTRINO PHYSICS AND NEUTRINO MASS
}

\author{
F. Boehm and P. Vogel \\ Physics Department, California Institute of Technology, Pasadena, \\ California 91125
}

\section{CONTENTS}

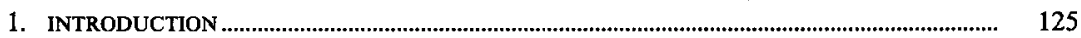

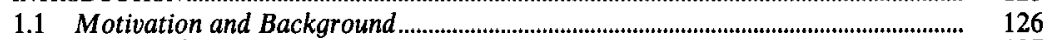

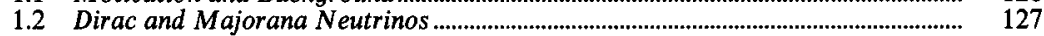

1.3 Neutrino Mixing and Oscillations ..................................................................... 128

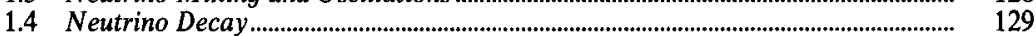

1.5 Summary of Present Experimental Evidence ……....................................................... 131

2. OSCILLATION EXPERIMENTS WITH REACTOR NEUTRINOS ..................................................... 132

2.1 The Detection Reaction .............................................................................................. 132

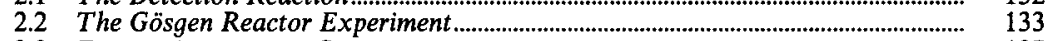

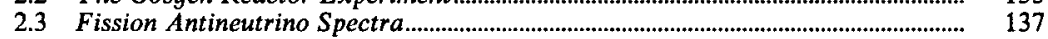

3. DIRECT AND INDIRECT NEUTRINO MASS MEASUREMENTS .................................................. 138

3.1 Electron Antineutrino Mass from Beta Decay Endpoints ......................................... 138

3.2 Muon and Tau Neutrino Mass Limits .................................................................. $\quad 140$

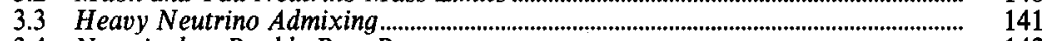

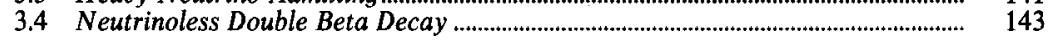

4. OUTLOOK

\section{INTRODUCTION}

Among the principal concerns in neutrino physics today are the questions of whether neutrinos are massive and, if so, whether the neutrinos emitted in a weak decay are pure or mixed quantum states. The concept of mixed neutrinos has been with us for more than 20 years, having first been introduced by Maki et al (1) and by Pontecorvo (2) following the demonstration in 1962 that more than one type (flavor) of neutrino existed. After having been dormant for some time, the interest in these issues was reborn in recent years with the advent of grand unified theories, which predict nonvanishing neutrino mass and which can accommodate neutrino 


\section{BOEHM \& VOGEL}

mixing in a natural way. Controversial experiments also refueled the excitment (and consternation) of researchers in this endeavor.

The field was reviewed by Bilenky \& Pontecorvo (3) in 1978, by Frampton \& Vogel (4) in 1982, and by Bullock \& Devenish (5) in 1983. Here we focus on recent developments in the phenomenology of low-energy neutrino physics to the extent that it provides information on neutrino mass and mixing. We discuss neutrino decay, experiments on neutrino oscillations, kinematic mass measurements, searches for heavy neutrino admixtures, and studies of neutrinoless double beta decay. Subjects not discussed are accelerator-based (high-energy) experiments, neutrinoelectron scattering, and certain other lepton-number-violating processes.

\subsection{Motivation and Background}

In the standard minimal electroweak theory, neutrinos are purely lefthanded and massless and their three distinct lepton numbers (electron, muon, tau) are conserved. Therefore observation of a neutrino mass and of neutrino mixing would signal new physics beyond the minimal standard model.

Numerous theoretical proposals incorporating finite neutrino mass have been presented. At the electroweak level a neutrino mass can be introduced by extension of the minimal model (6-8), but it is often difficult to explain why neutrinos are so much lighter than the other fermions. Grand unified theories, based on various larger symmetry groups, can accommodate small neutrino masses in the range of $10^{-6}-10^{+1} \mathrm{eV}(9-11)$. Scaling of the neutrino mass is often expected (the tau neutrino is the heaviest and the electron neutrino the lightest); the masses are then proportional to the first or second power of the corresponding charged-lepton (or quark) masses.

Neutrino mass in the $\mathrm{eV}$ range has dramatic cosmological and astrophysical ramifications. Based on the universally accepted hot big bang model, one predicts, in analogy with the $3-\mathrm{K}$ microwave background, a background for each light $\left(m_{v}<1 \mathrm{MeV}\right)$ stable neutrino, with the number density of $n_{v}+n_{\bar{v}} \approx 110 \mathrm{~cm}^{-3}$. In that case there are about $10^{10}$ times as many neutrinos as baryons, and neutrinos heavier than about $1 \mathrm{eV}$ could dominate the total mass of the universe. From observation of the present expansion rate of the universe, one obtains an upper limit for the total average mass density that translates into the condition $\sum m_{v}<200 \mathrm{eV}$ (see, for example, 12), where the summation is over all flavors of light stable neutrinos. The only assumption used in deriving this upper limit is that the cosmological constant vanishes (13).

Massive neutrinos could become gravitationally bound to galaxies or galactic clusters. In that case neutrinos of $m \approx 10 \mathrm{eV}$ would account for the apparent large excess of the dark matter over the luminous matter $(14,15)$. 
Massive neutrinos would also play an important role in the theory of formation and development of inhomogeneities in the universe, leading to superclusters, clusters, and individual galaxies (16).

Several proposals have been made for the detection of the background neutrino sea. Recent analysis $(17,18)$ shows, however, that all of them lead to immeasurably small effects even when we include the possible local density enhancement of up to $10^{5}$ due to the gravitational binding of the neutrinos.

The "solar neutrino puzzle" is often mentioned in connection with the neutrino oscillation problem. In the experiment by Davis et al (19) based on the ${ }^{37} \mathrm{Cl}\left(v, \mathrm{e}^{-}\right)^{37} \mathrm{Ar}$ reaction with the $814-\mathrm{keV}$ threshold only $1 / 4$ to $1 / 3$ of the expected neutrino flux (20) is observed. Maximum oscillations among three neutrino flavors with a wavelength less than the Sun-Earth distance would indeed reduce the flux of the electron neutrinos reaching the earth by the factor of three. Alternative explanations of this puzzle, however, are not exhausted (21).

\subsection{Dirac and Majorana Neutrinos}

Massive charged leptons, such as electron, muon, or tau, are easily distinguished from their antiparticles. They are described by fourcomponent spinors and there is only one Lorentz-invariant and chargeconserving expression possible for the Lagrangian mass term:

$$
L_{\mathrm{D}}=m_{\mathrm{D}} \bar{\psi} \psi
$$

This mass term obviously conserves the lepton number.

The situation is more complicated for neutral fermions, such as neutrinos, because Lorentz invariance alone also allows another mass term

$$
L_{\mathrm{M}}=m_{\mathrm{M}}\left(\bar{\psi}^{\mathrm{c}} \psi+\bar{\psi} \psi^{\mathrm{c}}\right) \text {, }
$$

where $\psi^{\mathrm{c}}$ is the charge-conjugated spinor. The term $L_{\mathrm{M}}$ changes a neutrino into an antineutrino and thus violates lepton-number conservation. Particles described by $L_{\mathrm{D}}$ are Dirac neutrinos (distinct from their antiparticles); those described by $L_{\mathrm{M}}$ are Majorana neutrinos (identical with their antiparticles). Only two components of the Majorana spinor are independent. A Dirac neutrino is formally a special case of two Majorana neutrinos with identical masses and opposite $C P$ eigenvalues (e.g. 22, 23).

The distinction between the Dirac and Majorana neutrinos becomes important only if $m \neq 0$ or if both left-handed and right-handed currents participate in weak interactions. In particular, the neutrinoless double beta decay, which violates lepton-number conservation, becomes possible for Majorana neutrinos. Most grand unified theories predict massive Majorana neutrinos. 


\subsection{Neutrino Mixing and Oscillations}

In the standard electroweak theory each charged lepton $\ell^{-}$has its lefthanded neutrino partner $v_{\ell}$. The neutrinos $v_{\ell}$ are weak eigenstates, but are not necessarily states with a definite mass. That means that the mass term discussed in Section 1.2 is generally not diagonal in $v_{\ell}$. One can define a unitary mixing matrix $U$,

$$
v_{\ell}=\sum_{i=1}^{N} U_{\ell i} v_{i}
$$

where $v_{i}$ are states of a definite mass (the mass term is diagonal in $v_{i}$ ), and $N \geq 3$ is the number of generations (flavors). It is customary to order the $v_{i}$ in such a way that $U_{i i}$ is as nearly diagonal as possible, and one can then use the approximate terms "electron neutrino mass," etc.

When matrix $U_{t i}$ is not exactly diagonal, we are led to the concept of neutrino oscillations. Let $v_{\ell}$ be created by weak charged-current reaction at $t=0$ with momentum $p$. The time development of such a state is given by

$$
v_{\ell}(t)=\sum_{i=1}^{N} U_{\ell i} \exp \left[-i\left(p+\frac{m_{i}^{2}}{2 p}\right) t\right]
$$

provided $p \gg m_{i}$.

The different components of Equation 4 have time-dependent phases leading to typical interference effects. In particular, the probability that one encounters a weak eigenstate $\ell^{\prime}$ after time $t$ equals

$$
P_{t}\left(\ell \rightarrow \ell^{\prime}\right)=\sum_{i=1}^{N}\left[\left|U_{\ell i}\right|^{2}\left|U_{\ell^{\prime} i}\right|^{2}+\sum_{j \neq i} U_{\ell i} U_{\ell j} U_{\ell^{\prime} i}^{*} U_{\ell^{\prime} j}^{*} \exp \left(-i \frac{m_{i}^{2}-m_{j}^{2}}{2 p}\right) t\right]
$$

that is, this probability is an oscillating function of time $t$, or of distance $L=c t$. Such an effect is called neutrino oscillation and requires nonvanishing, nondegenerate neutrino masses, and at least some nondiagonal matrix elements in $U$.

Study of oscillations does not furnish the neutrino masses themselves but the quantity $\Delta m^{2}=\left|m_{j}^{2}-m_{i}^{2}\right|$, obtained from the wavelength

$$
L_{\mathrm{osc}}(m)=\frac{2.5 \times E(\mathrm{MeV})}{\left|m_{i}^{2}-m_{j}^{2}\right|(\mathrm{eV})^{2}}
$$

associated with each pair of $i, j$ neutrinos with masses $m_{i}, m_{j}$. Mixing coefficients $U_{t i}$ are obtained from the oscillation amplitudes. Note that the oscillation pattern depends on $L / E$, the ratio of the distance to the neutrino energy. In an experiment both the $L$ dependence and the $E$ dependence can be used to explore oscillations. 
Neutrino oscillations described above are "flavor" oscillations; the electron, muon, etc numbers are no longer conserved, but their sum (the total lepton number) is still conserved. Neutral-current weak interactions are not affected by the "flavor" oscillations. Observation of such oscillations would mean that at least some neutrinos are massive; no distinction between Dirac and Majorana neutrinos could be made, however.

For Majorana neutrinos, oscillations of the "second" class are possible (24); they also affect the neutral current and violate the total lepton number. In such a case a neutrino beam can produce antileptons (and an antineutrino beam can produce leptons). The probability of such a $\Delta L=2$ process is, however, suppressed by the helicity factor $\left(m_{v} / E\right)^{2}$ and becomes essentially unobservable $(4,25)$. Processes with $\Delta L=2$ also become possible if weak interactions explicitly involve right-handed lepton currents, as discussed in Section 3.5.

\subsection{Neutrino Decay}

If neutrinos have mass, the heavier ones could decay into the lighter ones. Neutrino decay has never been seen; however, if it were observed it would give information on the masses, and, because at least two neutrino flavors are involved, on neutrino mixing.

The radiative decays

$$
\begin{aligned}
& v_{2} \rightarrow v_{1}+\gamma \\
& v_{2} \rightarrow v_{1}+\mathrm{e}^{+}+\mathrm{e}^{-}
\end{aligned}
$$

are generally considered the most likely candidates (26-28). The photon mode (Equation 7) has its rate suppressed by the factor $\left(m_{\ell} / m_{\mathrm{W}}\right)^{4}\left(m_{\ell}\right.$ is the charged-lepton mass, $m_{\mathrm{W}}$ is the $\mathrm{W}$ mass; these particles appear as intermediate states in the corresponding Feynman graphs), and for the Dirac neutrino one obtains

$$
\Gamma_{\gamma}=\frac{\alpha}{2}\left[\frac{3 G_{\mathrm{F}}}{32 \pi^{2}}\right]^{2} m_{v_{2}}^{5}\left|\sum_{\ell} U_{\ell 2} U_{\ell 1}^{*} \frac{m_{\ell}^{2}}{m_{\mathrm{W}}^{2}}\right|^{2} .
$$

Substituting the tau mass for $m_{\ell}$ we find

$$
\Gamma_{\gamma} \approx\left(10^{29} \text { years }\right)^{-1}\left(\frac{m_{v_{2}}}{30 \mathrm{eV}}\right)^{5}\left|U_{\tau 1} U_{\tau 2}^{*}\right|^{2}
$$

Various proposals to speed up the decay have been discussed (e.g. 26, 29); they involve a very heavy fourth lepton and other assumptions. The decay rate of Majorana neutrinos is more difficult to calculate (29).

In a laboratory experiment one tries to observe the decay of moving, usually highly relativistic, neutrinos. The laboratory decay rate $\Gamma^{\mathrm{Lab}}$ is 
130 BOEHM \& VOGEL

related to the invariant center-of-mass rate by the time dilatation factor:

$$
\Gamma^{\mathrm{Lab}}=\frac{m_{v}}{E_{v}} \Gamma^{\mathrm{CM}}
$$

An experiment thus furnishes a value, or an upper limit, of the product $m_{v} \Gamma_{\gamma}$. Existing experimental limits are much poorer than the theoretical estimates of Equation 9. For electron neutrinos one obtains (30)

$$
m_{v_{e}} \Gamma_{y} \leq 3 \times 10^{-3} \mathrm{eV} / \mathrm{s} \text {, }
$$

which is about $10^{35}$ times greater than Equation $9 \mathrm{a}$ for $m_{v}=30 \mathrm{eV}$ and maximum mixing. Nevertheless, the corresponding decay length exceeds the Sun-Earth distance by a factor of $10^{5}$, excluding decay as a possible explanation of the solar neutrino puzzle. For muon neutrinos the corresponding limit is $m_{v} \Gamma_{\gamma}<0.11 \mathrm{eV} / \mathrm{s}(31)$.

The decay into the electron-positron pair (Equation 8) can proceed only when $m_{v}>2 m_{\mathrm{e}}$. Its rate is much faster (28) than the photon emission mode,

$$
\Gamma_{\mathrm{ee}}=\frac{G_{\mathrm{F}}^{2}}{192 \pi^{2}} m_{v_{2}}^{5}\left|U_{\mathrm{e} 2} U_{\mathrm{e} 1=v_{\mathrm{e}}}^{*}\right|^{2} h\left(\frac{m_{\mathrm{e}}}{m_{v_{2}}}\right),
$$

where $h$ is a phase-space factor such that $h(0)=1, h(0.5)=0$. We know from the neutrino oscillation searches described below that

$$
\left|U_{\mathrm{e} 2} U_{\mathrm{e} 1=v_{\mathrm{e}}}\right|^{2} \leq 0.05
$$

and thus

$$
\Gamma_{\mathrm{ee}} \leq\left(6 \times 10^{5} \mathrm{~s}\right)^{-1}\left(\frac{m_{v_{2}}}{1 \mathrm{MeV}}\right)^{5} .
$$

The experimental and theoretical limits for neutrino lifetime are summarized in Figure 1.

Other decay modes, such as $v \rightarrow 3 v^{\prime}$ have also been considered but are typically even slower or their description involves additional assumptions.

Astrophysical considerations allow one to exclude certain neutrino mass-lifetime combinations independently of the theoretical decay rates $(12,32)$. Such considerations use available data on the microwave and diffuse photon backgrounds, supernova energetics and emission, etc. As described for example by Turner (12), one is left with only three allowed "corners" of the neutrino mass-lifetime space: long-lived light neutrinos $\left(m_{v}<200 \mathrm{eV}, \tau>10^{22} \mathrm{~s}\right.$ for the maximum mass), long-lived heavy neutrinos $\left(m_{v}>1 \mathrm{GeV}, \tau>10^{24} \mathrm{~s}\right)$, and short-lived heavy neutrinos $\left(m_{v}>10 \mathrm{MeV}, \tau<10^{2} \mathrm{~s}\right.$ for the minimal mass). The neutrino lifetime boundaries based on astrophysical arguments are also shown in Figure 1. 


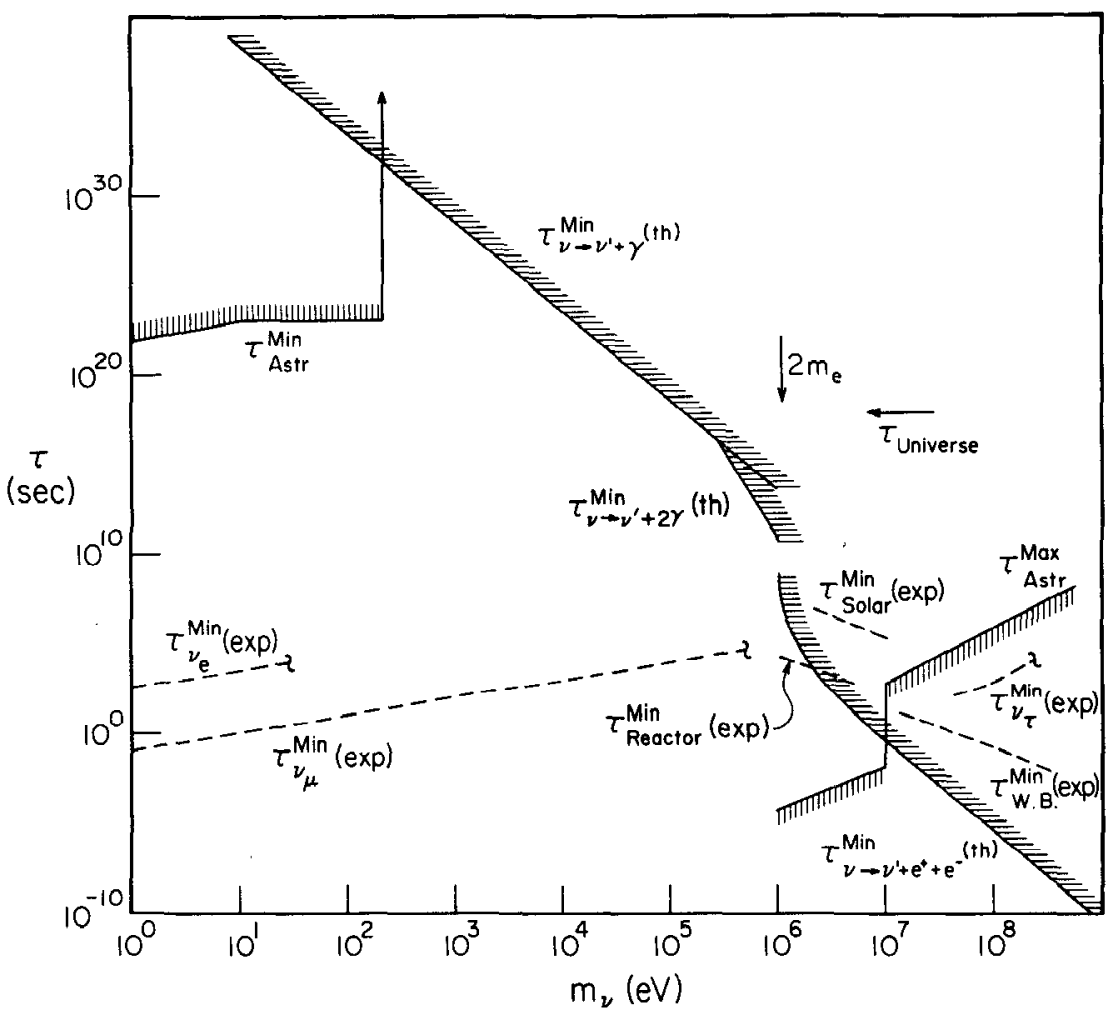

Figure 1 Theoretical and experimental limits of neutrino lifetime: $\tau($ th) is the lifetime of Equations 9 and 10 for maximum mixing, where one assumes that $\tau$ is the heaviest charged lepton; the horizontally shaded regions are allowed. The theoretical lifetime for $2 \gamma$ decay (96) is also shown. The $\tau_{\text {Astr }}$ are boundaries based on astrophysical arguments (12); vertically shaded regions are allowed. Experimental lower limits for the dominantly coupled neutrinos extend to the upper limits of the corresponding mass: $\tau_{v_{o}}(30), \tau_{v_{\mu}}(31), \tau_{v_{\tau}}$ (33). The terms $\tau_{\text {Solar }}$ (34), $\tau_{\text {Reactor }}(95)$, and $\tau_{\text {w.B. }}(33)$ are limits for less strongly coupled heavy neutrinos.

\subsection{Summary of Present Experimental Evidence}

To date there is no confirmed evidence that neutrinos have finite mass. A reported deviation in the beta decay endpoint in ${ }^{3} \mathrm{H}$, if confirmed, may yet indicate a mass in the range of $20-30 \mathrm{eV}$. Oscillation experiments with lowenergy neutrinos from a reactor provide an upper limit for the mass parameter $\Delta m^{2}=\left|m_{2}^{2}-m_{1}^{2}\right| \leq 0.016 \mathrm{eV}^{2}$, if we assume the maximum value of the mixing strength $\sin ^{2} 2 \theta$, as well as an upper limit of $\sin ^{2} 2 \theta \leq 0.16$ for $\Delta m^{2}>0.05 \mathrm{eV}^{2}$. As to admixtures from heavy $(>1 \mathrm{keV})$ neutrinos, experiments involving two- and three-body decays provide limits of about 


\section{BOEHM \& VOGEL}

$10^{-2}$ to $10^{-3}$ for the strength of admixture of $m_{v}=1-100 \mathrm{keV}$ neutrinos, and still better limits for neutrinos with $m_{v}>100 \mathrm{keV}$. If neutrinos are Majorana particles, lepton-number-violating double beta decay may occur. This process has not been observed at the present level of sensitivity, which leads to the conclusion that neutrino mass cannot exceed a value in the range of 5-16 eV, or that right-handed weak currents cannot contribute more than $2-6 \times 10^{-5}$ with respect to the left-handed current. The smallness (or absence) of neutrino mass is consistent with cosmological bounds confining the heaviest neutrino to masses of less than $200 \mathrm{eV}$. The solar neutrino puzzle is not inconsistent with the absence of oscillations in laboratory experiments, and may still indicate oscillations with large mixing and small $\left(<0.01 \mathrm{eV}^{2}\right)$ mass parameters.

\section{OSCILLATION EXPERIMENTS WITH REACTOR NEUTRINOS}

\subsection{The Detection Reaction}

A number of experiments to study neutrino oscillations have been carried out with low- and high-energy neutrinos $(5,35)$. So far, no evidence for neutrino oscillations has been found. With this in mind, a simple analysis in terms of two neutrino states is therefore appropriate. This description includes two parameters only, a mixing angle $\theta\left(\sin \theta=U_{12}=-U_{21}\right.$; $\left.\cos \theta=U_{11}=U_{22}\right)$ and a mass parameter $\Delta m^{2}=\left|m_{2}^{2}-m_{1}^{2}\right|$.

Low-energy experiments involving electron neutrinos are of the disappearance type since not enough energy is available to create the muon or tau lepton. Thus the probability for a $\vec{v}_{\mathrm{e}}$ having disappeared by undergoing oscillations into another neutrino state (inclusive reaction) is given by the deviation from unity of $P(\ell \rightarrow \ell)=1-P\left(\ell \rightarrow \ell^{\prime}\right)$, where $P\left(\ell \rightarrow \ell^{\prime}\right)$ can be written for our case, following Equation 5 , as

$$
P\left(\ell \rightarrow \ell^{\prime}\right)=\frac{\sin ^{2} 2 \theta}{2}\left[1-\cos \frac{2.53 \times \Delta m^{2}(\mathrm{eV})^{2} \times L(m)}{E_{v}(\mathrm{MeV})}\right] .
$$

The disappearance experiments thus test the effect of all oscillation channels (independent of the assumption of only two neutrino states). These experiments are sensitive to a large range of $\Delta m^{2}$; in particular, the lowest $\Delta m^{2}$ is obtained for low $E_{v}$ values.

Nuclear reactors are powerful sources of electron antineutrinos because the fission fragments are unstable and undergo a series of beta decays. The antineutrinos thus are emitted with energies characteristic of nuclear beta decay. A neutrino detector positioned at varying distances from a reactor is capable of measuring the $\bar{v}_{\mathrm{e}}$ yield as a function of energy and position, 
thus allowing one to verify both the $E$ and $L$ dependence of oscillations (Equation 11).

To detect the low-energy antineutrinos, the inverse neutron decay (36), $\bar{v}_{\mathrm{c}} \mathrm{p} \rightarrow \mathrm{e}^{+} \mathrm{n}$, is well suited. This reaction, which can be identified by a timecorrelated positron and neutron signature, has a cross section that is a function of the outgoing positron energy, given by

$$
\sigma\left(E_{\mathrm{e}}\right)=\frac{2 \pi^{2} \hbar^{3}}{m_{\mathrm{e}}^{5} c^{7} f \tau_{\mathrm{n}}} p_{\mathrm{e}} E_{\mathrm{e}}
$$

where $\tau_{\mathrm{n}}$ is the neutron mean life and $f$ is the usual statistical function including the Coulomb correction for $Z=1$. The outgoing positron and incoming antineutrino energies are related through

$$
E_{\bar{v}}=E_{\mathrm{e}}+\left(M_{\mathrm{n}}-M_{\mathrm{p}}\right) .
$$

According to Wilkinson (37), $f=1.6857$ and the recommended adjusted average neutron lifetime is $\tau_{\mathrm{n}}=900 \pm 9 \mathrm{~s}$. However, it should be noted that not all experimental data on $\tau_{\mathrm{n}}$ are mutually consistent.

In an experiment at a nuclear reactor one actually measures the positron yield, which is (assuming no oscillations) given by

$$
Y\left(E_{\mathrm{e}}\right) \sim \sigma\left(E_{\mathrm{e}}\right) \mathrm{n}\left[E_{\bar{v}}=E_{\mathrm{e}}+\left(M_{\mathrm{n}}-M_{\mathrm{p}}\right)\right],
$$

where $n\left(E_{\bar{v}}\right)$ is the reactor antineutrino flux per unit energy.

With the present good accuracy $(<5 \%)$ in the experimental yield it is necessary to consider higher order terms in $\sigma$, such as neutron recoil corrections, weak magnetism, radiative corrections (bremsstrahlung), and higher order Coulomb terms (38).

Besides the $\bar{v}_{\mathrm{e}} \mathrm{p} \rightarrow \mathrm{e}^{+} \mathrm{n}$ reaction, the charged-current reaction on the deuteron, $\bar{v}_{e} \mathrm{~d} \rightarrow \mathrm{e}^{+} \mathrm{nn}$, and the competing neutral-current reaction, $v_{e} \mathrm{~d}$ $\rightarrow \bar{v}_{\mathrm{e}} \mathrm{pn}$, have been studied, the latter being insensitive to oscillations. Based on the ratio of the total neutron yields in these reactions Reines et al (39) found indication for neutrino oscillation. In a later paper (40), however, the values were modified and the evidence for oscillations is no longer statistically significant.

\subsection{The Gösgen Reactor Experiment}

The Caltech-Munich-SIN group has conducted two experiments at the Gösgen reactor in Switzerland; one (41) with the detector at a distance of $38 \mathrm{~m}$ from the core and another one (42) at $46 \mathrm{~m}$. Prior to these experiments, a measurement was made at the ILL reactor in Grenoble (France) at a distance of $8.7 \mathrm{~m}$ using a similar detector (43). The setup of the Gösgen experiment is sketched in Figure 2. The neutrinos were detected by the 
A Annual Reviews

www.annualreviews.org/aronline

\section{BOEHM \& VOGEL}

reaction $\bar{v}_{\mathrm{e}} \mathrm{p} \rightarrow \mathrm{e}^{+} \mathrm{n}$ using a composite liquid scintillation detector and ${ }^{3} \mathrm{He}$ multiwire proportional chambers. A time-correlated $\mathrm{e}^{+}, \mathrm{n}$ event constituted a valid signature.

Pulse shape discrimination in the scintillation counter has proved to be a powerful technique for eliminating correlated neutron background events. These events are caused by cosmic-ray-induced fast neutrons recoiling on protons in the liquid scintillation counter. The recoil gives rise to a scintillation counter trigger, followed, after a thermalization period, by a neutron capture signal in the ${ }^{3} \mathrm{He}$ counter. Neutrons associated with the reactor are entirely absent in these experiments.

About 11,000 neutrino-induced events were recorded in 6-9 months of reactor-on time. Backgrounds for each position were recorded during a one-month reactor-off period. Figure 3 shows the difference spectrum of reactor-on minus reactor-off for both positions, together with a curve representing the expected spectrum for no oscillations. The latter was obtained from the on-line beta spectroscopic measurements at the ILL reactor by Schreckenbach et al (44) studying ${ }^{235} \mathrm{U}$ and ${ }^{239} \mathrm{Pu}$ fission targets. These two isotopes account for about $89 \%$ of the total fission energy at the Gösgen reactor. The remaining $11 \%$ are due to fission of ${ }^{238} \mathrm{U}$ and ${ }^{241} \mathrm{Pu}$.

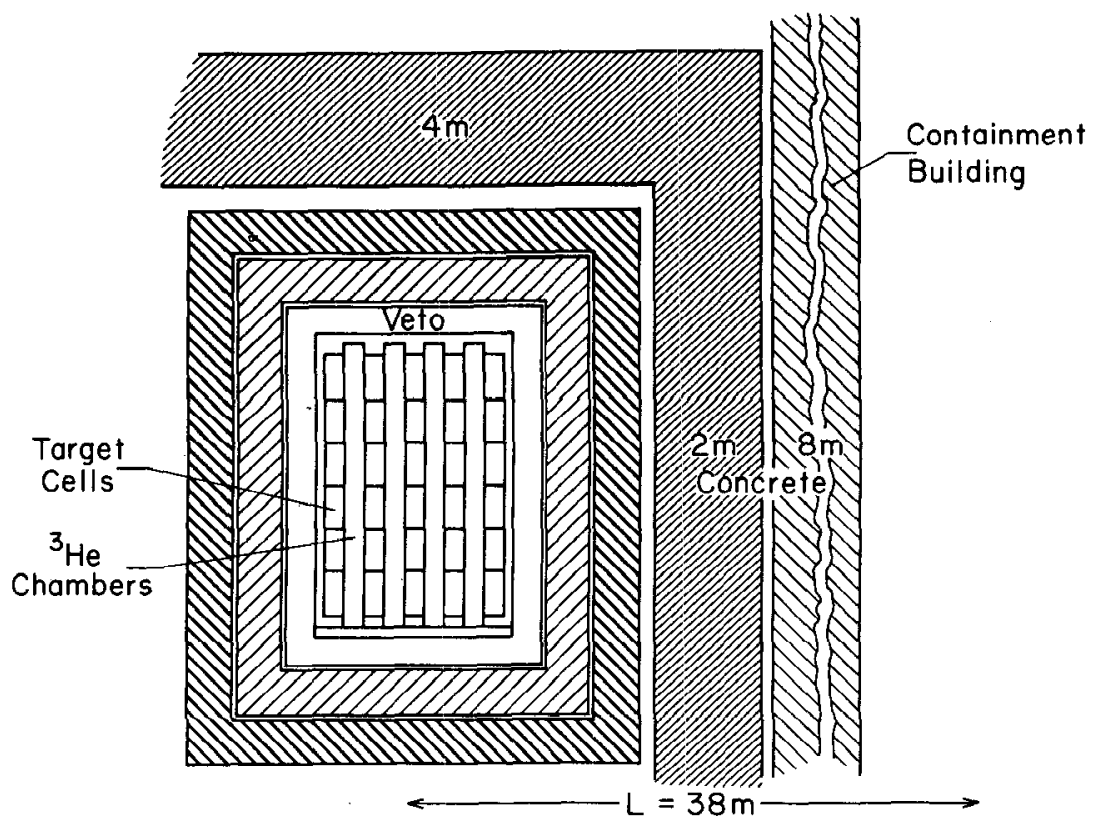

Figure 2 Experimental setup of the neutrino detector at the Gösgen reactor. (The drawing is not to scale.) 
The calculations of Vogel et al (45) were used to evaluate the contribution to the antineutrino spectra from ${ }^{238} \mathrm{U}$ and ${ }^{241} \mathrm{Pu}$. The variation in time of the contributions of each fissioning isotope is well known and was taken into account.

In Figure 4 the observed yields for the $38-\mathrm{m}$ and $46-\mathrm{m}$ positions at Gösgen $(41,42)$, and the 8.7-m position at ILL (43) are displayed, in units of the no-oscillation yield, as a function of $L / E_{v}$. As can be seen in Figures 3 and 4 , there is good agreement between experiment and the expectation for no oscillations.

The data at two or three positions can also be analyzed without resorting to the no-oscillation spectrum. The exclusion plots of Figure 5 were obtained by considering the ratios of the data at $8.7,38$, and $46 \mathrm{~m}$ for each energy bin and fitting them to calculated ratios for various oscillation parameters. A $\chi^{2}$ test to all possible values of $\Delta m^{2}$ and $\sin ^{2} 2 \theta$ resulted in the $90 \%$ confidence limits $(\mathrm{CL})$ displayed in Figure 5 . The analysis leading to the dashed curve in Figure 5 is entirely independent of the no-oscillation neutrino spectrum as well as the detector efficiency calibration. It has been concluded that there are no neutrino oscillations with parameters larger than those contained to the right of the curves in Figure 5.

Finally, an important question should be addressed: Where in the $\Delta m^{2}$ vs $\sin ^{2} 2 \theta$ plane should one continue to search for oscillations? Unfortunately, there is no guidance whatsoever from theory. As to the

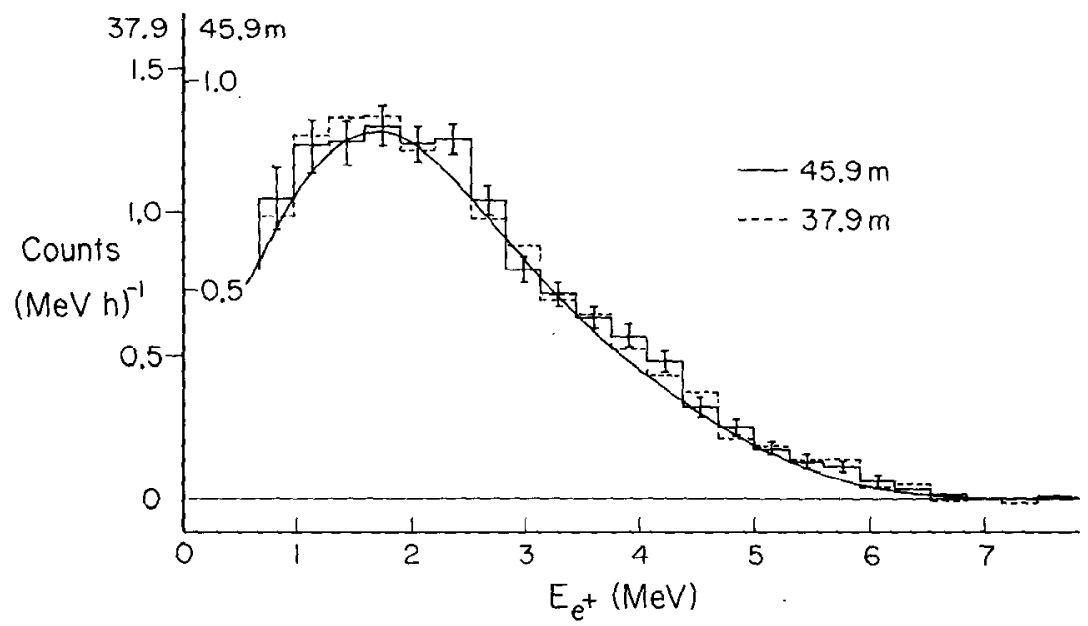

Figure 3 Results of the Gösgen experiments. Positron spectra obtained by subtracting reactor-off from reactor-on spectra for the $38-\mathrm{m}$ and $46-\mathrm{m}$ experiments. The energy bin is $0.305 \mathrm{MeV}$. The solid curve represents the predicted positron spectrum assuming no neutrino oscillations. 
136 BOEHM \& VOGEL

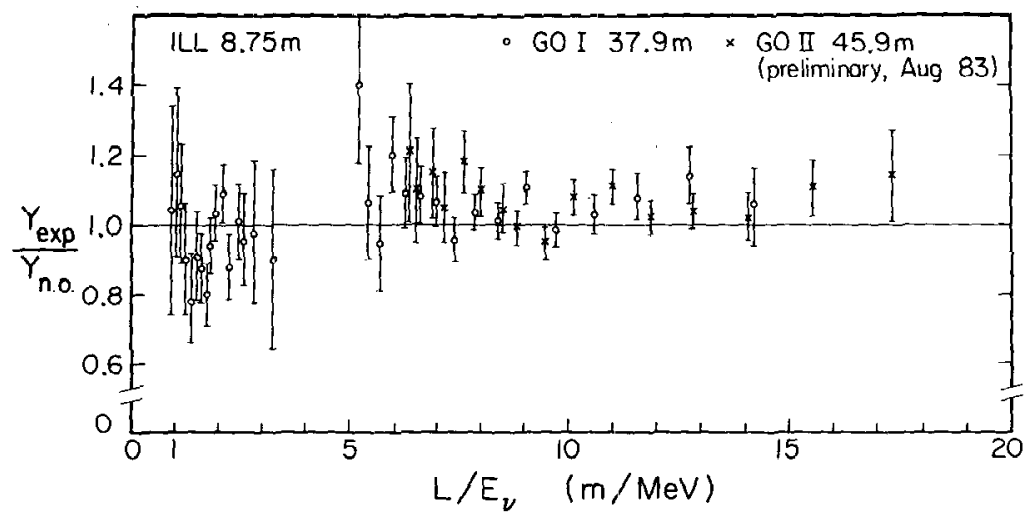

Figure 4 Ratio of experimental to predicted (for no oscillations) positron spectra at $8.7,38$, and $46 \mathrm{~m}$ from the reactor core. The errors of the data points shown are statistical (from 46 ).

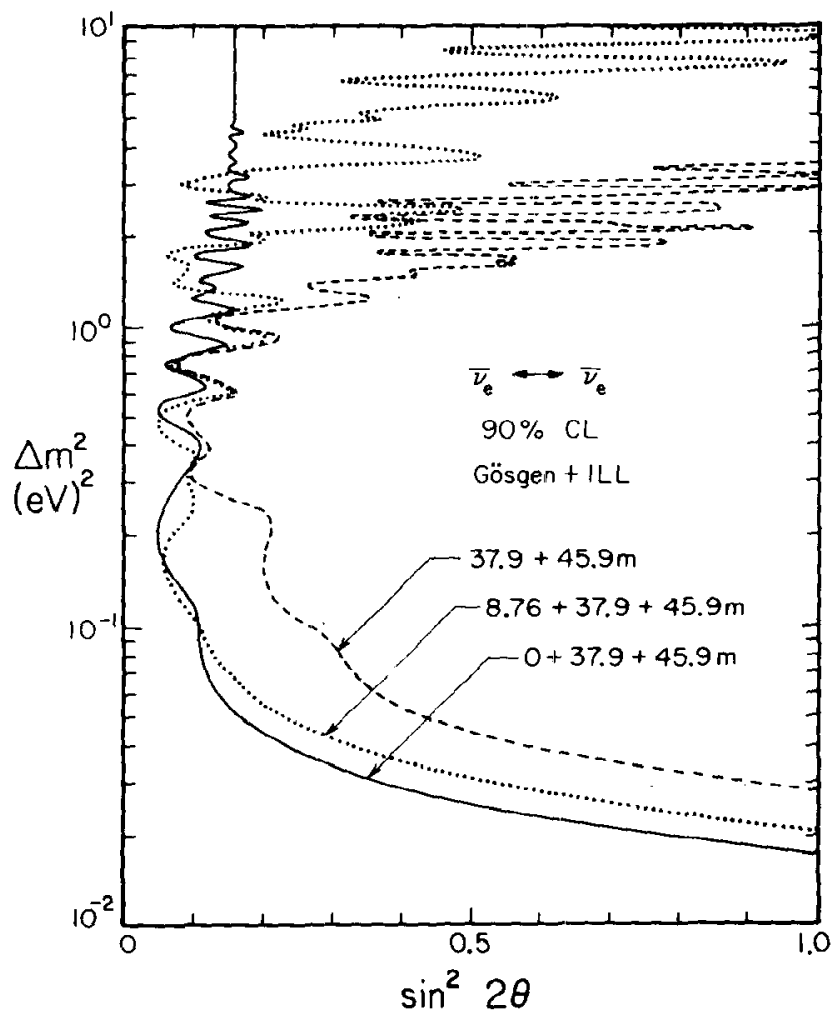

Figure 5 Exclusion plot obtained from the Gösgen and ILL experiment at $90 \%$ confidence limit. The curve labelled $37.9+45.9 \mathrm{~m}$ refers to the limit obtained by using the $37.9-\mathrm{m}$ and 45.9-m data only, for example. The $0 \mathrm{~m}$ label represents the on-line beta spectroscopic measurement (see text). 


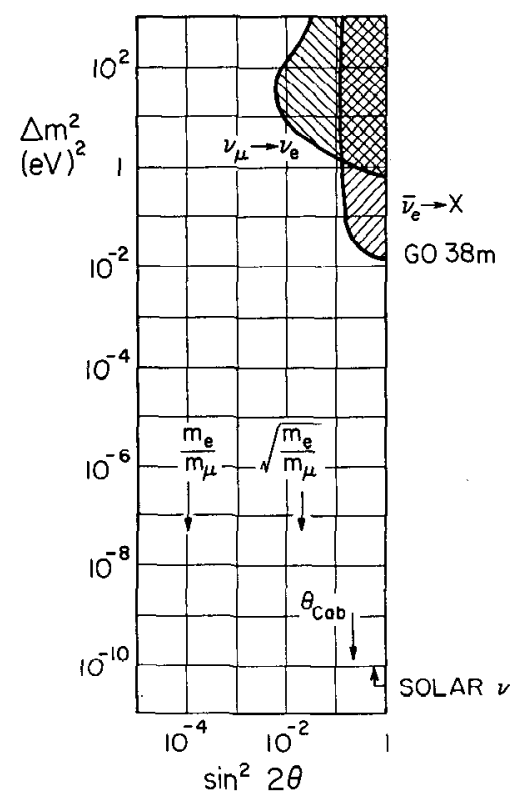

Figure 6 Expanded $\Delta m^{2}$ vs $\sin ^{2} 2 \theta$ plane showing current experimental limits and some dimensional guesses.

mixing angle, we can state that present limits are smaller than the Cabibbo angle. Figure 6 shows these limits, together with other possible dimensional guesses (lepton mass ratios). If the solar neutrino experiments are indeed telling us that neutrinos oscillate with large mixing angle, the $\Delta m^{2}$ values must lie between $10^{-2}$ and $10^{-10} \mathrm{eV}^{2}$, a region increasingly difficult to explore.

\subsection{Fission Antineutrino Spectra}

The above analysis of the reactor oscillation experiments is based on comparison of the positron yields measured at different distances and is therefore independent of the reactor antineutrino flux. Knowledge of this flux is, however, still important because it allows one to correct for the (weak) time dependence of the reactor fuel composition and for the (small) contribution of the ${ }^{238} \mathrm{U}$ and ${ }^{241} \mathrm{Pu}$ fission, and most importantly it makes it possible to determine the maximal allowed mixing angle for large $\Delta m^{2}$ (Figure 5).

Because in each beta decay both $\mathrm{e}^{-}$and $\bar{v}_{\mathrm{e}}$ are emitted with correlated energies, one can deduce the $\bar{v}_{\mathrm{e}}$ spectrum from the experimentally determined electron spectrum. For the main reactor fuels, ${ }^{235} \mathrm{U}$ and ${ }^{241} \mathrm{Pu}$, the electron spectra have been determined $(44,47)$ very accurately for 


\section{BOEHM \& VOGEL}

kinetic energies up to $\sim 7 \mathrm{MeV}$ (and somewhat less accurately up to $8 \mathrm{MeV}$, where the spectrum is more than 1000 times weaker than its maximum).

When the electron spectrum is converted into the antineutrino spectrum, an uncertainty of $4-6 \%$ at $90 \% \mathrm{CL}$ is introduced (47). It is important at this level of accuracy to correct for deviations from the allowed beta decay spectrum shape (38).

The reactor $\bar{v}_{\mathrm{e}}$ spectrum can also be obtained by adding spectra of all beta branches of all fission fragments (see, for example, 45). This method, although in principle straightforward, requires knowledge of fission yields, beta decay $Q$ values, and branching ratios. For short-lived fission fragments this information is often uncertain or unavailable and one must resort to nuclear structure considerations. Thus the uncertainties in the resulting spectrum are typically larger than those obtained from conversion of the experimental electron spectra.

\section{DIRECT AND INDIRECT NEUTRINO MASS MEASUREMENTS}

In this section we review the results of experiments on neutrino mass based on observation of charged particles emitted in weak decays. The momenta and energies of the charged particles, as well as the overall decay rate, are affected by the neutrino mass and mixing. A detailed account of the theory of weak decays with massive mixed neutrinos has been given by Shrock (48).

It is useful to distinguish between dominantly and subdominantly admixed neutrinos. While dominantly admixed neutrinos can be treated as constituting the principal mode, for both massive and massless neutrinos, weakly admixed neutrinos can be regarded as an additional decay channel open only for massive and mixed neutrinos. Neutrinoless double beta decay based on a measurement of the electron spectrum is also included in this section.

\subsection{Electron Antineutrino Mass from Beta Decay Endpoints}

In nuclear beta decay, as in any three-body decay $\mathrm{x} \rightarrow \mathrm{y}+\ell+\bar{v}_{\ell}$, there may be several endpoint energies $E_{\mathrm{Max}}^{i}$ each associated with a neutrino mass $m_{v_{i}}$. They are related to each other by

$$
E_{\mathrm{Max}}^{i}=\frac{M_{\mathrm{x}}^{2}+m_{\ell}^{2}-\left(M_{\mathrm{y}}+m_{v_{i}}\right)^{2}}{2 M_{\mathrm{x}}} .
$$

The corresponding Kurie plot $[N(E) / p E F(Z, E)]^{1 / 2}$ (or its analog) has kinks at each endpoint and an infinite slope at the maximal endpoint (minimal $m_{v}$ ). In the neighborhood of each endpoint the neutrino is nonrelativistic; hence deviations of the spectrum from the shape corresponding to massless neutrinos are linear in $m_{v}$. 
Here we discuss the dominant mode associated with a single neutrino with mass $m_{v}$. The nucleus ${ }^{3} \mathrm{H}$ has the desirable features of a low $Q$ value (to enhance the relative number of decays near the endpoint and to decrease the required relative resolution) and a reasonably short lifetime (to increase specific activity).

Recent results on the ${ }^{3} \mathrm{H}$ beta decay are summarized in Table 1 . There is evidence for a nonvanishing electron antineutrino mass from the ITEP experiments $(51,53)$ but independent confirmation of this important result has yet to come forward.

One of the main problems in the ${ }^{3} \mathrm{H}$ experiments is the effect of the spectrometer resolution and response. In the mentioned experiments the resolution has been comparable or larger than the value or limit of $m_{v}$. In the neighborhood of the endpoint the finite spectrometer resolution $R$ causes the slope of the Kurie plot to decrease, whereas the finite neutrino mass $m_{v}$ causes it to increase. Thus an error $\Delta R$ in $R$ results in an assignment of a fictitious neutrino mass related to the true mass $m_{v}$ by

$$
\left(m_{v}^{\mathrm{fic}}\right)^{2} \approx 2 \Delta R \cdot R+m_{v}^{2} \text {. }
$$

An accurate knowledge of the full resolution function, including the effects of electron energy losses in the source, is therefore crucial. It is also necessary to take into account the natural width of the calibration lines in the determination of the spectrometer resolution function, as pointed out by Simpson (54).

Another problem, affecting the interpretation of the results, deals with the fate of the spectator electron originally bound to the ${ }^{3} \mathrm{H}$ atom or molecule. After the sudden change of the nuclear charge this electron does not end up in a single stationary quantum state. In particular, the

Table $1 \quad \bar{v}_{\mathrm{c}}$ mass determination in ${ }^{3} \mathrm{H}$ decay

\begin{tabular}{lcccc}
\hline Author, Year (Ref.) & Source & $\begin{array}{c}\text { Resolution } \\
(\mathrm{eV})\end{array}$ & $m_{\bar{v}}(\mathrm{eV}), \mathrm{CL}$ & $E_{0}{ }^{\mathrm{d}}(\mathrm{eV})$ \\
\hline Bergkvist, 1972 (49) & ${ }^{3} \mathrm{H}$ in Al & 50 & $<55,90 \%$ & $18,623 \pm 16^{\mathrm{c}}$ \\
Tretjakov et al, 1976(50) & valine $^{\mathrm{a}}$ & 45 & $<33,90 \%$ & $18,575 \pm 13$ \\
Lyubimov et al, 1980(51) & valine & 45 & $14 \leq m_{\bar{v}} \leq 46,{ }^{\mathrm{b}} 99 \%$ & $18,577 \pm 13$ \\
Simpson, 1981 (52) & ${ }^{3} \mathrm{H}$ in Si (Li) & 220 & $<60,95 \%$ & $18,543 \pm 5^{\mathrm{f}}$ \\
Boris et al, 1983 (53) & valine & 20 & $33 \pm 1.1,{ }^{\mathrm{c}} 60 \%$ & $18,583.2 \pm 0.3^{\mathrm{c}}$ \\
\hline
\end{tabular}

\footnotetext{
${ }^{a} 3 \mathrm{H}$-tagged $\mathrm{C}_{5} \mathrm{H}_{11} \mathrm{NO}_{2}$.

${ }^{b}$ Limits include different final ${ }^{3} \mathrm{He}$ states. When the neglected intrinsic widths of the calibration lines are taken into account, the lower limit of $m_{\bar{v}}=14 \mathrm{eV}$ is reduced to zero (53).

${ }^{\circ}$ For the theoretical final state of valine as calculated in (55); if a full range of final states is considered, one obtains model-independent lower limits of $m_{\bar{v}} \geq 20 \mathrm{eV}, E_{Q} \geq 18,575 \mathrm{eV}$ at $95 \% \mathrm{CL}$ (53).

${ }^{d}$ For ${ }^{3} \mathrm{He}^{+}$ground state.

${ }^{c}$ Corrected by $13.5 \mathrm{eV}$ to transform from the measured average excitation energy.

${ }^{\mathrm{f}}$ Corrected by $24 \mathrm{eV}$ to transform from the measured atomic mass difference.
} 
probability of finding the final ${ }^{3} \mathrm{He}$ ion in its ground state is only $0.6-0.7$, depending on the chemical composition of the source, and the average excitation energy of the final state is $13-20 \mathrm{eV}$. The spectrum of final states can be reliably calculated for the free ${ }^{3} \mathrm{H}$ atom. This spectrum was computed for valine by Kaplan et al (55). Clearly this problem should be considerably alleviated if the resolution could be made smaller than the expected excitation energy of the final atomic or molecular complex.

The background level determines the minimal distance from the endpoint, that is, the maximal electron energy, where data still have statistical significance. There has been a significant reduction, by a factor of about 20 , in the background level of the recent ITEP experiment (53) over the results of Lyubimov et al (51).

While the values of $m_{v}$ in Table 1 are mutually consistent, the endpoints $E_{0}$ are not. The value of $E_{0}$ has been determined independently by Smith et al (56) by measurement of the ${ }^{3} \mathrm{H}-{ }^{3} \mathrm{He}$ atomic mass difference. The resulting value of $E_{0}=18549 \pm 7 \mathrm{eV}$ is in a significant disagreement with the ITEP result (53). It was recently pointed out (57) that the results of (53) imply that the true endpoint of the electron spectrum (i.e. where the spectrum would end if the resolution were a delta function) is $18580-33 \approx 18550 \mathrm{eV}$, in agreement with (56) but at the same time indicating vanishing (or very small) neutrino mass.

The previous discussion dealt with the antineutrino mass. Similar studies of positron decays, and thus of the electron neutrino mass, are difficult because electron capture $(\mathrm{EC})$ dominates over positron emission at low decay energies. Information on the electron neutrino mass could be extracted from the study of the endpoint region of inner bremsstrahlung accompanying electron capture (58). The bremsstrahlung arises at low energies mainly from p-capture, and its intensity is enhanced by the resonant process if the energy is not far from some $\mathrm{p} \rightarrow \mathrm{s} \mathrm{x}$-ray transition $(58,59)$. Tests $(60)$ in the EC decay of ${ }^{193} \operatorname{Pt}(Q=56.6 \pm 0.3 \mathrm{keV})$ set an upper limit of $m_{v_{e}}<500 \mathrm{eV}$ for the electron neutrino mass. Electron capture in ${ }^{163} \mathrm{Ho}[Q=2.58 \pm 0.10 \mathrm{keV}(61)$, and $T=4570 \pm 50$ years (62)] has attracted considerable interest as a candidate for further study; other possibilities are ${ }^{158} \mathrm{~Tb}(63)$ and ${ }^{157} \mathrm{~Tb}(64)$. At the present time the study of bremsstrahlung accompanying EC is considerably less sensitive to neutrino mass than the study of the electron spectrum in ${ }^{3} \mathrm{H}$ decay.

\subsection{Muon and Tau Neutrino Mass Limits}

In two-body decays, such as the pion decay $\pi \rightarrow \mu+v_{\mu}$, a value or an upper limit for the muon neutrino mass can be determined from kinematics. Again we assume that only one neutrino mass eigenstate is dominantly coupled to the muon. 
In the pion rest frame the muon neutrino mass is related to the dominant (maximal) muon momentum by the quadratic dependence

$$
m_{v_{\mu}}^{2}=m_{\pi}^{2}+m_{\mu}^{2}-2 m_{\pi}\left(p_{\mu}^{2}+m_{\mu}^{2}\right)^{1 / 2} \text {. }
$$

Thus in order to obtain a neutrino mass value it is necessary to determine with sufficient accuracy the muon momentum and pion and muon masses.

The study of the pion decay in flight allows one to reduce the absolute value of the neutrino momentum in the laboratory frame and thus increase sensitivity to the neutrino mass; the results are also less dependent on the precise knowledge of the pion mass.

At the present time both methods lead to virtually identical upper limits on the muon neutrino mass. In the pion decay at rest one obtains $m_{v_{\mu}}<0.52 \mathrm{MeV}(90 \% \mathrm{CL})$ using the pion momentum determined by Daum et al (65) and the pion mass of Lu et al (66). A slightly better limit of $0.49 \mathrm{MeV}(90 \% \mathrm{CL})$ was achieved recently (67). In the pion decay in flight experiment (68) the limit is $m_{v_{\mu}}<0.50 \mathrm{MeV}(90 \% \mathrm{CL})$ and the uncertainty is dominated by the systematic errors in the determination of $p_{\mu}-p_{\pi}$ for the forward going muons ( $p_{\pi} \approx 350 \mathrm{MeV} c^{-1}$ ).

In decays having three particles in the final state, one can take advantage of the regime where the neutrino has a small momentum (see the discussion of beta decay above). However, until now only the study of the $K_{\mu_{3}}$ decay (69) led to a meaningful limit $m_{v_{\mu}}<0.65 \mathrm{MeV}(90 \% \mathrm{CL})$.

Finally, to connect to cosmological and astrophysical considerations discussed in Sections 1.1 and 1.4, we note that the above limits of the muon neutrino mass are in the middle of the "forbidden" region.

The experimental limits on the tau neutrino mass are quite poor. Limits of $m_{v_{t}}<250 \mathrm{MeV}(95 \% \mathrm{CL})$ were obtained by Bacino et al (70) and by Blocker et al (71).

\subsection{Heavy Neutrino Admixing}

We now explore the possibility that one or more heavy neutrinos may be admixed to a light neutrino state. Here we again assume that the weak interaction eigenstates $v_{t}$ are superpositions of mass eigenstates, $v_{i}$ (Equation 3). Experimental evidence points to the fact that a state $v_{\ell}$ (such as $v_{\mathrm{e}}$ ) is predominantly composed of one light neutrino $v_{i}$ (such as $v_{1}$ ). If heavy neutrinos exist, their admixture must therefore be small. In this case $\left|U_{\ell, 2 \text { or } 3}\right| \ll 1$ and a decay proceeding via the heavy neutrinos has a branching ratio proportional to $\left|U_{\epsilon i}\right|^{2}$.

The two-body decays $K \rightarrow \pi v, \pi \rightarrow \mu \nu$, or $\pi \rightarrow$ ev offer sensitive tests to study these branches (48). In the lepton spectrum each mass eigenstate is expected to manifest itself as a monochromatic peak at some energy below the regular lepton peak associated with the light neutrino, with an intensity 


\section{BOEHM \& VOGEL}

proportional to $\left|U_{\ell i}\right|^{2}$. Several spectroscopic experiments aimed at finding these secondary peaks have been carried out, but no evidence for a heavy neutrino decay has been reported so far. The best current limits for the mixing strength $\left|U_{\ell i}\right|^{2}$ from these experiments for admixtures to electron neutrinos $(\ell=\mathrm{e})$ and muon neutrinos $(\ell=\mu)$ are summarized in Figure 7. References are provided in the caption.

The study of three-body decays also lends itself to the search for heavy neutrino branches (48). As mentioned above, in nuclear beta decay a heavy neutrino would show up as a discontinuity in the electron spectrum, for example. Recent studies by Schreckenbach et al (73) of the electron spectra of ${ }^{64} \mathrm{Cu}$ and by Simpson (72) of the spectrum of ${ }^{3} \mathrm{H}$ provide limits for neutrino mixing in the mass range of $1 \mathrm{keV}$ to $0.5 \mathrm{MeV}$ (see Figure 7).

Limits for $\left|U_{C i}\right|^{2}$ from oscillation experiments are also shown in Figure 7. For $v_{\mathrm{e}} \rightarrow \mathrm{x}$ we use $\left|U_{\ell i}\right|^{2}=(1 / 4) \sin ^{2} 2 \theta$, with $\sin ^{2} 2 \theta<0.16(42)$; existing results from $v_{\mu} \rightarrow \mathrm{X}$ are not relevant in the mass region shown.

As mentioned in Section 1.4, the mass region between $200 \mathrm{eV}$ and $10 \mathrm{MeV}$ is forbidden for stable or unstable neutrinos based on astrophysical and cosmological arguments.
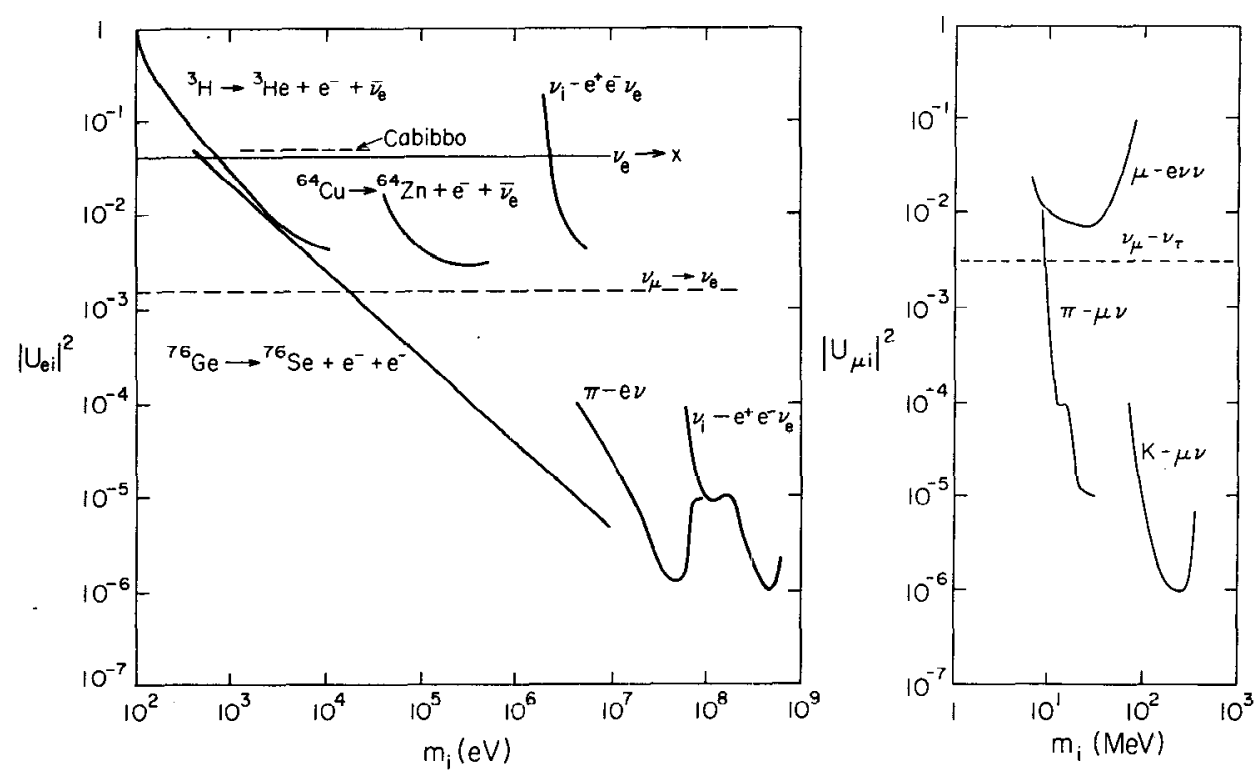

Figure 7 Limits for mixing coefficients $\left|U_{\mathrm{e} i}\right|^{2}$ and $\left|U_{\mu i}\right|^{2}$ describing the admixture of heavy neutrinos to the electron neutrino and the muon neutrino, respectively. The regions above the curves are excluded. The curves are based on the following references: ${ }^{3} \mathrm{H} \rightarrow{ }^{3} \mathrm{He}+\mathrm{e}^{-}+\bar{v}(72),{ }^{64} \mathrm{Cu} \rightarrow{ }^{64} \mathrm{Zn}+\mathrm{e}^{-}+\bar{v}(73),{ }^{76} \mathrm{Ge} \rightarrow{ }^{76} \mathrm{Se}+\mathrm{e}^{-}$ $+\mathrm{e}^{-}$(74), $\pi \rightarrow \mathrm{e} v$ (75), $\nu \rightarrow \mathrm{eev}(33,95), \mu \rightarrow \mathrm{evv}(48), \pi \rightarrow \mu v(77), \mathrm{K} \rightarrow \mu \nu$ (78). 


\subsection{Neutrinoless Double Beta Decay}

A sensitive source of information for neutrino mass and right-handed currents is the neutrinoless double beta decay, a semileptonic weak process of second order. Double beta decay proceeds from a nucleus $Z$ to $Z+2$ and should become observable if the first-order process $Z$ to $Z+1$ is energetically forbidden. There are two types of double beta decay: the twoneutrino decay, $Z \rightarrow(Z+2)+\mathrm{e}_{1}+\bar{v}_{1}+\mathrm{e}_{2}+\bar{v}_{2}$, and the zero-neutrino decay, $Z \rightarrow(Z+2)+\mathrm{e}_{1}+\mathrm{e}_{2}$. The former is expected to occur from standard theory; its study is of interest since it might help in estimating the value of the nuclear matrix elements needed to analyze the second process. The zero-neutrino process, if observed, would signal violation of lepton-number conservation, which can be associated with nonzero Majorana neutrino mass or right-handed weak currents $(79,80)$.

Figure 8 illustrates both the $2 v$ and the $0 v$ processes. The characteristic signature of the latter is a monochromatic peak in the spectrum of the total electron energy $\varepsilon_{0}$. The $0 v$ decay could proceed by virtual neutrino exchange, as illustrated in the figure. This is a two-nucleon mechanism: a neutron $n_{1}$ emits an electron $e_{1}$ and a neutrino, and the latter is absorbed by a neutron $n_{2}$, which then emits an electron $e_{2}$. The process can only proceed
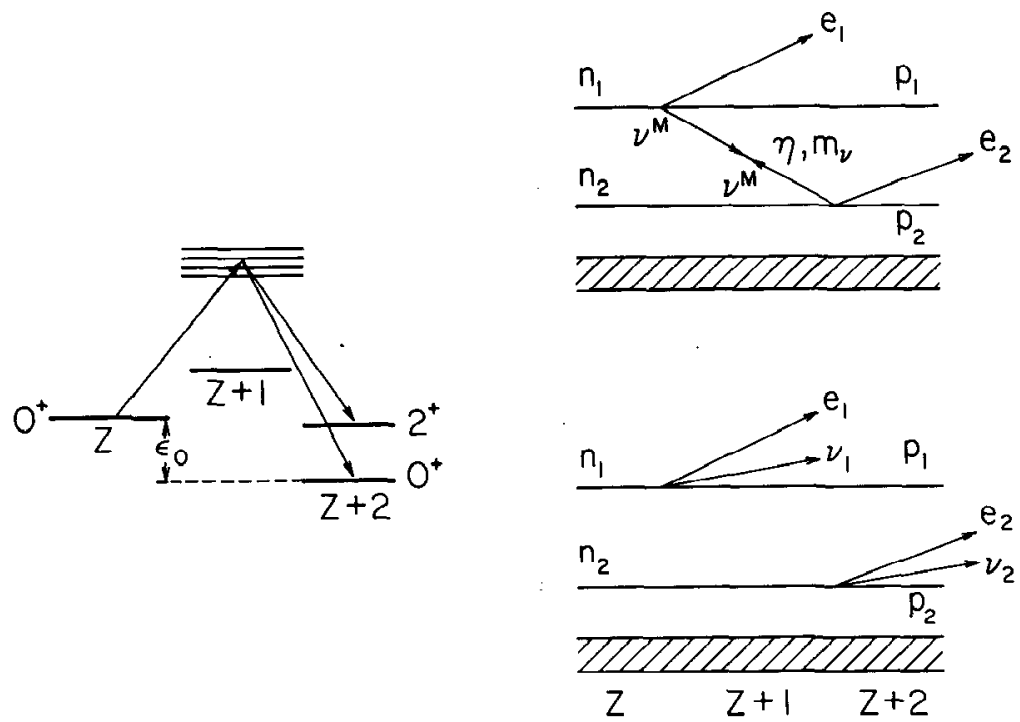

Figure 8 Illustration of double beta decay: (top right) neutrinoless, (bottom right) twoneutrino. 


\section{4 ВOEHM \& VOGEL}

if the neutrino is a Majorana particle $\left(v^{\mathrm{M}} \equiv \bar{v}^{\mathrm{M}}\right)$. In addition, in the standard theory angular momentum conservation prevents the $0 v$ process from proceeding since only purely right-handed antineutrinos can be emitted, and only purely left-handed neutrinos can be absorbed. Two mechanisms have been identified $(79,80)$ that violate the perfect helicity of the neutrino and thus allow the $0 v$ process to proceed:

1. The charged lepton current in the weak interaction has an explicit right helicity admixture given by

$$
j_{\lambda} \approx \mathrm{e} \gamma_{\lambda}\left[\left(1+\gamma_{5}\right)+\eta\left(1-\gamma_{5}\right)\right] v^{\mathrm{M}} \text {. }
$$

We assume here that the hadronic current has its standard form. (In the standard theory $\eta=0$ and $m_{v}=0$, hence the subscript $M$ in Equation 17 is not necessary, as explained in Section 1.2.)

2. The neutrino has a mass so that a "wrong" helicity amplitude $\sim m_{v} / E$ arises even though the interaction is purely "left-handed."

In the discussion below we assume that neutrinoless double beta decay can be described by the above two parameters, the right-handed current parameter $\eta$ and the Majorana neutrino mass $m_{v}$. Other descriptions have been presented in literature, including "quasi-Dirac" neutrinos or the existence of more than one neutrino (81). As to the nuclear aspect, it is assumed that the two-nucleon process sketched above dominates the decay probability and far outweighs the $\mathrm{N}^{*}$ mechanism (80). In calculating the rate, the summation over the nuclear intermediate states is carried out in the closure approximation.

TRANSITION PROBABILITIES The $2 v$ process can proceed with massless Dirac or Majorana neutrinos and its rate is given for $0^{+} \rightarrow 0^{+}$transitions by

$$
\Gamma^{2 v} \approx F_{2 v}\left(\varepsilon_{0}\right) C\left(\varepsilon_{0}, Z\right)\left(\left|M_{\mathrm{GT}}\right|^{2} /\left\langle\Delta E_{\mathrm{N}}+\frac{\varepsilon_{0}}{2}+m_{\mathrm{e}}\right\rangle^{2}\right),
$$

where $F_{2 v}\left(\varepsilon_{0}\right)$ is the 4-fermion phase-space factor; $C\left(\varepsilon_{0}, Z\right)$ is the Coulomb function; $M_{\mathrm{GT}}$ is the appropriate second-order Gamow-Teller-type matrix element $\left\langle f\left\|\sigma^{(1)} \cdot \sigma^{(2)} \tau_{ \pm}^{(1)} \tau_{ \pm}^{(2)}\right\| i\right\rangle$ describing the virtual transitions via states in $Z+1$ (see Figure 8); and $\Delta E_{\mathrm{N}}$ is the average nuclear energy difference between these states and the initial state. The main uncertainty in this decay rate stems from $\left|M_{\mathrm{GT}}\right|^{2}$ and may be as large as a factor of $10^{ \pm 2}$. A rough estimate for the $2 v$ half-life yields $T_{1 / 2}^{2 v} \approx 10^{22 \pm 2} \mathrm{y}$.

A similar estimate can be made for the $0 v$ process. Both right-handed current (RHC) and mass mechanisms have been considered (79). For a 
transition between ground states $\left(0^{+} \rightarrow 0^{+}\right)$this rate is given by

$$
\Gamma^{0 v} \approx F_{0 v}\left(\varepsilon_{0}\right) C\left(\varepsilon_{0}, Z\right)\left|\frac{M_{\mathrm{GT}}}{\left\langle r_{i j}\right\rangle m_{\mathrm{p}}}\right|^{2} .
$$

The quantity $F_{0 v}\left(\varepsilon_{0}\right)$ is the 2-fermion phase-space factor; it contains terms in neutrino mass $\left(m=m_{v} / m_{\mathrm{e}}\right)$ and in $\mathrm{RHC}(\eta)$ in the following form,

$$
F_{0 v}\left(\varepsilon_{0}\right)=m^{2} f_{m}\left(\varepsilon_{0}\right)+m \eta f_{m \eta}\left(\varepsilon_{0}\right)+\eta^{2} f_{\eta}\left(\varepsilon_{0}\right)
$$

The term $f_{m}$ has the same energy dependence as in allowed beta decay (s-wave), and $f_{\eta}$ has higher powers in energy reflecting the momentum transfer dependence in the RHC process. The quantity | | in Equation 19 represents the Gamow-Teller matrix element divided by an average nucleon separation distance $\left\langle r_{i j}\right\rangle$ measured in proton Compton wave length $1 / m_{\mathrm{p}}$. The $\left\langle r_{i j}\right\rangle$ appears because the virtual neutrino is exchanged between two nucleons within the same nucleus. Here a rough estimate gives $T_{1 / 2}^{0 v} \approx 10^{15 \pm 2} \eta^{-2}$ (or $m^{-2}$ ) y.

We note that $0 v$ decay is $\sim 10^{7}$ times faster than the corresponding $2 v$ decay if $\eta=1$ or $m=1$. Part of this difference can be understood if in Equations 17 and 18 one retains only the leading $\varepsilon_{0}$ powers and omits all (non-numerical) common factors. This yields $\varepsilon_{0}^{4} / \Delta E_{N}^{2}$ for $\Gamma^{2 v}$ and $\left(m_{\mathrm{p}} /\left\langle r_{i j}\right\rangle m_{\mathrm{p}}\right)^{2}$ for $\Gamma^{0 v}$. Using $\left\langle r_{i j}\right\rangle m_{\mathrm{p}} \approx \Delta E_{\mathrm{N}} / m_{\mathrm{e}}\left(m_{\mathrm{p}}\right.$ was in fact introduced into Equation 19 in view of this), one obtains a factor of $\sim 10^{4}$ in the ratio of the rates. The remaining difference stems from numerical factors in $F_{0 v}$ and $F_{2 v}$.

Selection rules could help distinguish between the mass and RHC mechanisms $(79,80)$. For the mass term, only $0^{+} \rightarrow 0^{+}$transitions are allowed, while for the RHC term, one can have $0^{+} \rightarrow 0^{+}, 1^{+}, 2^{+}$. (This has to do with the additional momentum transfer dependence of the transition amplitude.) Thus, if a $0^{+} \rightarrow 2^{+}$branch were observed, it would give evidence for right-handed currents.

DISCUSSION OF EXPERIMENTAL EVIDENCE Double beta decay has been studied in several nuclei both by geochemical techniques [extraction of the daughter $(Z+2)$ from the parent $(Z)$ in an old ore] and with counters. Geochemical experiments cannot, of course, distinguish between $0 v$ and $2 v$ decays. We discuss below the results for some selected cases that are particularly suited for sensitive tests. For a more complete review see, for example, (81).

Te ratio In calculating the rates for double beta decay, the largest uncertainty stems from the nuclear matrix element $M_{\mathrm{GT}}$. The idea of eliminating the matrix elements by comparing two isotopes presumed to 
have similar nuclear structure is therefore attractive. The ratio of the halflives is then given by the ratio of the phase-space factors $F_{2 v}$ and $F_{0 v}$ for the two isotopes. Such a comparison has been made for ${ }^{128} \mathrm{Te}$ and ${ }^{130} \mathrm{Te}$. In the $2 v$ process, one expects $T_{1 / 2}^{130}(2 v) / T_{1 / 2}^{128}(2 v)=1.8 \times 10^{-4}$. For the $0 v$ process, this ratio is always larger and its exact value depends on the parameters $m_{v}$ and $\eta$. The geochemical work by Kirsten et al (82) gives $T_{1 / 2}^{130} / T_{1 / 2}^{128}=$ $(1.0 \pm 1.1) \times 10^{-4}$, in agreement with the $2 v$ prediction and compatible with the absence of $0 v$ decay. The upper limits for $m_{v}$ and $\eta$ (ignoring neutrino mixing) are $m_{v}<5 \mathrm{eV}$ and $\eta<2 \times 10^{-5}$, independently of the specifics of the calculation, aside from the assumed equality of the matrix elements. The ratio of Kirsten et al, however, disagrees with another geochemical ratio, obtained by Hennecke et al $(83), T_{1 / 2}^{130} / T_{1 / 2}^{128}=(6.3+0.2) \times 10^{-4}$. This result could be interpreted as requiring $0 v$ decay to occur and thus would constitute evidence for lepton-number violation with either $m_{v} \approx 10 \mathrm{eV}$ or $\eta \approx 5 \times 10^{-5}$. Clearly, no strong case can be made for or against leptonnumber nonconservation until this discrepancy is resolved.

${ }^{130} \mathrm{Te}$ The half-life of the decay of ${ }^{130} \mathrm{Te} \rightarrow{ }^{130} \mathrm{Xe}$, averaged over the existing geochemical experiments (84) (with large scatter of the data) is $2.6 \times 10^{21} \mathrm{y}$. Interpreted as $2 v$ decay, this yields a nuclear matrix element $M_{\mathrm{GT}}(2 v)=0.24(80)$. Haxton et al (85) calculated this decay and find $M_{\mathrm{GT}}(2 v)=3.0$ in the same units, i.e. a lifetime of $T_{1 / 2}^{2 v} \approx 1.7 \times 10^{19} \mathrm{y}$, about 150 times shorter than the observed one. It should be noted that the geochemical determinations depend critically on the so-called gas retention age of the minerals. Any error in this age would result in an increase in the deduced half-life with respect to the true one.

Notwithstanding the disagreement between the predicted and observed half-lives and setting aside the fact that additional assumptions are needed to relate the nuclear matrix elements for $2 v$ and $0 v$ decays, we shall obtain a crude estimate of $m_{v}$ based on the hypothesis that some fraction of the decay is due to a $0 v$ process induced by a finite $m_{v}$. Since this fraction is unknown we assume that it is $50 \%$, and furthermore that the ratio of the relevant matrix elements is that calculated by Haxton et al. This set of assumptions yield $m_{v}=130 \mathrm{eV}$; clearly, if the fraction due to $0 v$ decays is less than half, a correspondingly lower value of $m_{v}$ is derived. These arguments are illustrated in Figure 9 and summarized in Table 2.

${ }^{82} \mathrm{Se}$ Geochemical measurements (84) provide an average lifetime value for double beta decay of about $1.5 \times 10^{20} \mathrm{y}$ (with an estimated uncertainty of about $10 \%$ ). Again, the theoretical prediction (85) disagrees with the geochemical lifetime, as summarized in Table 2 and Figure 10. On the other hand, a cloud chamber experiment (87) gives a $2 v$ lifetime 15 times shorter than the geochemical one; however, with relatively large uncertainty. The 


$$
{ }^{130} \mathrm{Te}
$$

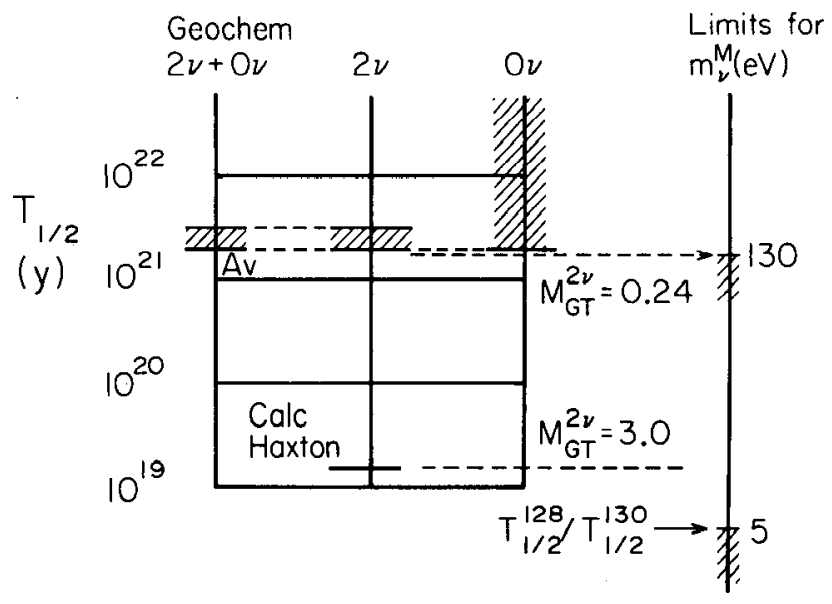

Figure 9 The geochemical and calculated half-lives and inferred neutrino mass limits for ${ }^{130} \mathrm{Te}$ (see text and Table 2 for explanations).

Table 2 Summary of selected double beta decay results

\begin{tabular}{|c|c|c|c|c|}
\hline & \multicolumn{2}{|c|}{ Experiment } & \multicolumn{2}{|c|}{ Calculation } \\
\hline & $\begin{array}{l}\text { Geochemistry } \\
\text { (Ref.) }\end{array}$ & $\begin{array}{l}\text { Laboratory } \\
\text { (Ref.) }\end{array}$ & $\begin{array}{c}\text { Doi et al } \\
\quad(80)\end{array}$ & $\begin{array}{l}\text { Haxton et al } \\
\quad(85,86)\end{array}$ \\
\hline \multicolumn{5}{|l|}{${ }^{76} \mathrm{Ge}$} \\
\hline $\begin{array}{l}\overline{T_{1 / 2}(2 v)(\mathrm{y})} \\
T_{1 / 2}(0 v)(\mathrm{y}) \\
m_{v}(\mathrm{eV})\end{array}$ & & $>3.7 \times 10^{22}(90)$ & $\begin{array}{c}2.3 \times 10^{21} \\
9.4 \times 10^{22 \mathrm{a}} \\
<16\end{array}$ & $\begin{array}{c}3.7 \times 10^{20} \\
<7\end{array}$ \\
\hline \multicolumn{5}{|l|}{${ }^{82} \mathrm{Se}$} \\
\hline $\begin{array}{l}\overline{T_{1 / 2}}(2 v)(\mathrm{y}) \\
T_{1 / 2}(0 v)(\mathrm{y}) \\
m_{v}(\mathrm{eV})\end{array}$ & $1.5 \times 20^{20}(84)$ & $\begin{array}{r}(1.0 \pm 0.4) \times 10^{19}(87) \\
>3: 1 \times 10^{21}(88)\end{array}$ & $\begin{array}{c}1.5 \times 10^{20} \\
3.2 \times 10^{22 \mathrm{a}} \\
\quad<33\end{array}$ & $\begin{array}{c}1.7 \times 10^{19} \\
<12\end{array}$ \\
\hline \multicolumn{5}{|l|}{${ }^{130} \mathrm{Te}$} \\
\hline 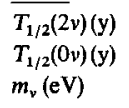 & $2.6 \times 10^{21 c}$ & & $\begin{array}{l}2.6 \times 10^{21} \\
2.5 \times 10^{23 a} \\
\quad<130\end{array}$ & $1.7 \times 10^{19}$ \\
\hline \multicolumn{5}{|l|}{${ }^{130 / 128} \mathrm{Te}$} \\
\hline $\begin{array}{l}\overline{T_{1 / 2}^{130 / 128}} \\
m_{v}(\mathrm{eV})^{\mathrm{b}}\end{array}$ & $(1.0 \pm 1.1) \times 10^{-4}(82)$ & & $<5$ & $<5$ \\
\hline
\end{tabular}

${ }^{\mathrm{a}}$ Assuming $m_{\mathrm{v}}=10 \mathrm{eV}$.

'Or $\eta<2 \times 10^{-5}$.

${ }^{c}$ Average value as quoted in (80). 


\section{BOEHM \& VOGEL}

experimental search for $2 v$ decay is continuing with an improved apparatus (89) and should help in clarifying the existing discrepancy. It is to be noted that for this transition there exists (88) an experimental limit $T_{1 / 2}^{0_{v}}>3.1$ $\times 10^{21} \mathrm{y}$. In analogy with the procedure applied to ${ }^{130} \mathrm{Te}$, we may assume, using this limit, that $\leq 5 \%$ of the geochemical rate is due to $0 v$ decay. With the same hypotheses as before, and dismissing the cloud chamber result, we obtain then $m_{v} \leq 32 \mathrm{eV}$. An alternative approach is to discard the geochemical result, and to rely on theoretical matrix elements, which agree, for the $2 v$ mode, with the cloud chamber result. This approach yields $m_{v}$ $\leq 12 \mathrm{eV}$.

${ }^{76} \mathrm{Ge}$ In the case of ${ }^{76} \mathrm{Ge}$ there now exist several sensitive laboratory results giving tight bounds on $T_{1 / 2}(0 v)$. Since there are no geochemical data from which to extract the matrix elements, one has to rely on the calculations by Haxton et al (86), possibly as modified by Doi et al (80). Using only the theoretical matrix elements of (86), and the best current laboratory limit (88) for the $0^{+} \rightarrow 0^{+}$transition of $T_{1 / 2}^{0 v}>3.7 \times 10^{22} \mathrm{y}$, one obtains $m_{v}<7 \mathrm{eV}$. Recalling the discrepancy, in the case ${ }^{130} \mathrm{Te}$ and ${ }^{82} \mathrm{Se}$ between the geochemical and theoretical $2 v$ rates, one may, following (80), "scale down" the theoretical matrix element by the factor corresponding to the discrepancy in ${ }^{82} \mathrm{Se}$ (the nuclide closer to ${ }^{76} \mathrm{Ge}$ ), and obtain $m_{v} \leq 16 \mathrm{eV}$ (see Table 2). The half-life limit for the $0^{+} \rightarrow 2^{+}$branch is $4 \times 10^{21}$ y (90).

A high-resolution Ge detector is an ideal instrument for obtaining

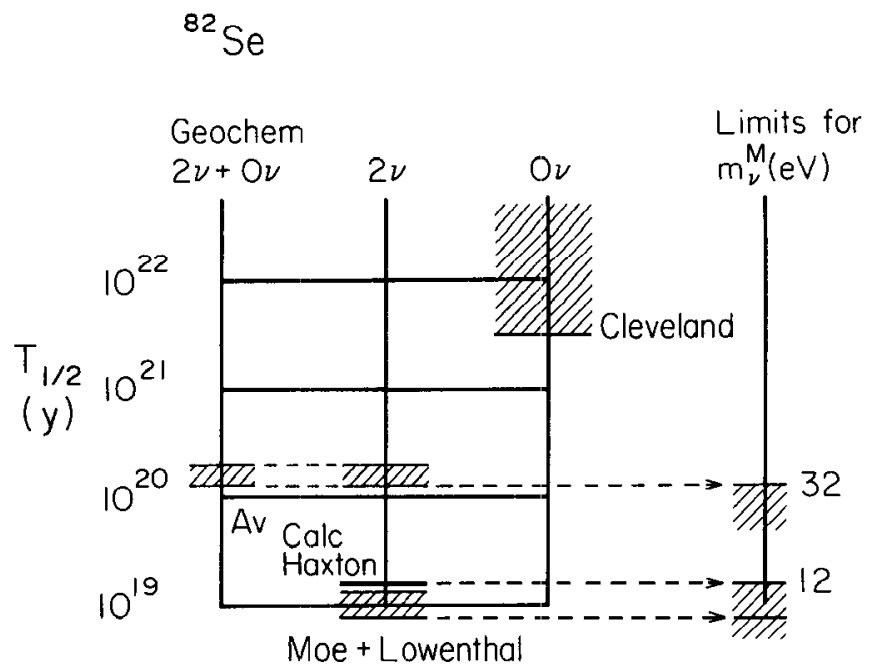

Figure 10 The geochemical and calculated half-lives and inferred neutrino mass limits for ${ }^{82} \mathrm{Se}$ (see text and Table 2 for explanations). 
sensitive limits for $0 v$ decay. ${ }^{76} \mathrm{Ge}$ occurs in germanium with a natural abundance of $7.8 \%$. Fiorini and his group pioneered the Ge experiments and the quoted best current upper limit has been reported by Bellotti et al (90) from an experiment in the Mont Blanc tunnel. Other laboratories (9193) have also reported results.

Figure 11 depicts the experimental arrangement of the Caltech (93) experiment. The Ge detector is shielded with $\mathrm{Cu}$ and $\mathrm{Pb}$ and surrounded by a radon tight can. A veto counter serves to reduce cosmic ray background.

The principal limitations for these experiments are detector size and, even more important, detector background. One of the principal sources of the background in the region of the decay energy $\varepsilon_{0}$ is the Compton contribution of the $2.6-\mathrm{MeV}$ gamma ray accompanying ${ }^{208} \mathrm{Tl}$ decay, a ubiquitous natural contamination. In the Caltech experiment (93) this contamination has been virtually eliminated. Other background components come from cosmic rays. They can be reduced by a veto system, as illustrated in Figure 11. However, high-energy bremsstrahlung and neutrons are not vetoed and to reduce these components one must install the experiment in an underground site, as Bellotti has shown.

Figure 12 illustrates a portion of the spectrum from the Caltech experiment. After $3820 \mathrm{~h}$ of running time there is no evidence for a peak at $2.04 \mathrm{MeV}$. From the number of counts, $N$, in a $3-\mathrm{keV}$ interval (the detector resolution) and its fluctuation, $\sqrt{N}$, one obtains a $1 \sigma$ limit for the $0 v$ lifetime of $T_{1 / 2}^{0 v}>1.9 \times 10^{22} \mathrm{y}$.

As to the future of the ${ }^{76} \mathrm{Ge}$ studies, it is safe to predict that ongoing efforts will stretch the sensitivity for $T_{1 / 2}^{0 v}$ to about $T_{1 / 2}^{0 v}>10^{23} \mathrm{y}$, which corresponds to a mass limit of $m_{v}<10 \mathrm{eV}$. To progress substantially below $10 \mathrm{eV}$, much larger sample sizes will be needed. The largest currently planned Ge experiments envision detectors of about $1000 \mathrm{~cm}^{3}$, or $3 \times 10^{24}$

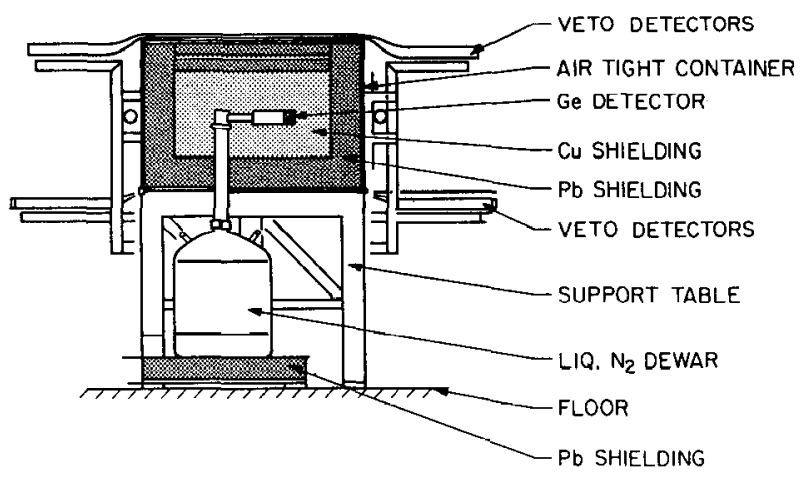

Figure 11 Ge detector setup for the Caltech double beta decay experiment. 


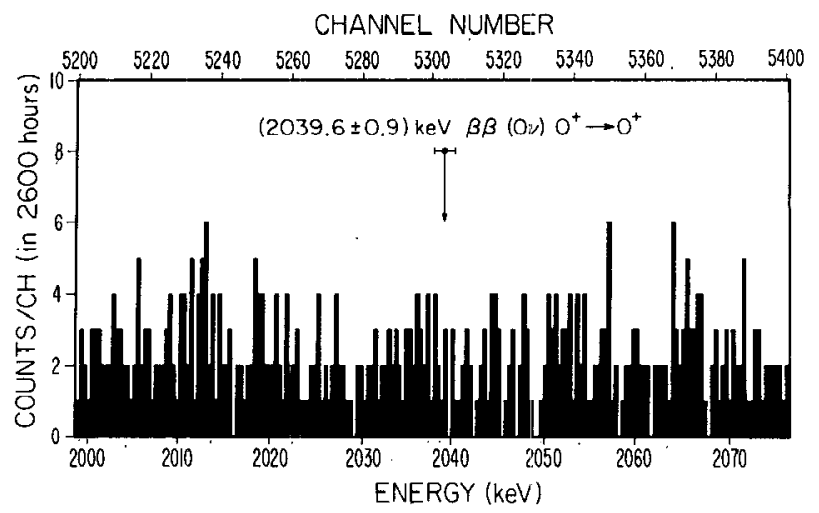

Figure 12 High-resolution Ge spectrum near total decay energy $\varepsilon_{0}=2040 \mathrm{keV}$.

atoms. In comparison, a large Xe time projection chamber (TPC) may have in excess of $10^{26}$ atoms of ${ }^{136} \mathrm{Xe}\left(\varepsilon_{0}=2.5 \mathrm{MeV}\right)$. Both liquid $\mathrm{Xe}(\mathrm{H}$. Chen, private communication) and pressured gas Xe (94) TPCs have been proposed. Depending on how well correlated electron tracks with energies up to $2.5 \mathrm{MeV}$ can be identified, these detectors may allow the exploration of neutrino mass down to $1 \mathrm{eV}$ or below.

\section{OUTLOOK}

Neutrino physics at low energies is capable of providing sensitive tests for neutrino mass and neutrino mixing in a manner complementary to the efforts in high-energy physics.

As to the $\bar{v}_{\mathrm{e}}$ mass, the recent much-publicized value of about $30 \mathrm{eV}$, if confirmed, would provide an important cornerstone for physics, astrophysics, and cosmology. Several independent experiments now underway to study the ${ }^{3} \mathrm{H}$ spectrum with improved resolution are therefore eagerly awaited.

Further progress in improving the sensitivity for inclusive oscillations of reactor neutrinos is expected to be slow, as it can only be accomplished with very much larger detectors. With a detector ten times larger, for example, the sensivitity for $\Delta m^{2}$ for full mixing may be as good as $0.005 \mathrm{eV}^{2}$, as compared to the present value of $0.016 \mathrm{eV}^{2}$, but the present mixing angle limit of $\sin ^{2} 2 \theta<0.16$, which is based on absolute flux measurements, cannot be improved significantly. On the other hand, progress is expected in the study of exclusive reactions, in particular in their sensitivity to small mixing angles. Work will also continue in the searches of small admixtures of heavy neutrinos. 
Double beta decay is one of the most promising topics having a clearly defined program for further study. The discrepancy between the geochemical and calculated lifetimes of the $2 v$ mode should be resolved, preferably by observing the $2 v$ mode in the laboratory. That would put the calculation of the nuclear matrix elements on a firmer basis and it would be possible to interpret with greater confidence the experimental lifetimes (or limits) for the $0 v$ mode in terms of the fundamental parameters of the neutrino Majorana mass or right-handed current. Independently of the uncertainty in the nuclear matrix element, the upper limit on $0 v$ decay provides a neutrino (Majorana) mass limit of $5-15 \mathrm{eV}$, which is already below the $30-\mathrm{eV}$ value derived from the ${ }^{3} \mathrm{H}$ experiment. Substantial improvements in sensitivity to $0 v$ double beta decay are expected with the advent of larger $\mathrm{Ge}$ detector arrays and Xe TPCs. These future experiments will probe neutrino mass down to about $1 \mathrm{eV}$ or below.

The solar neutrino puzzle remains unsolved. An independent determination of the solar neutrino flux, in particular of the low-energy neutrinos from the $\mathrm{p}+\mathrm{p} \rightarrow \mathrm{d}+\mathrm{e}^{+}+v_{\mathrm{e}}$ reaction, would help in deciding whether the problem has anything to do with neutrino oscillations.

Last but not least, progress in the predictive power of the underlying particle theory is urgently needed. Guidance as to the expected range of neutrino mass and mixing angles would help reinforce the enthusiasm of experimentalists. It would also help in integrating the problems of neutrino mass and mixing into the broader context of the fundamental properties of the constituents of matter.

\section{ACKNOWLEDGMENT}

This review was initiated at the Aspen Center for Physics; its hospitality and stimulating atmosphere are appreciated. Enlightening discussions with V. L. Telegdi are gratefully acknowledged. The work was supported by the US Department of Energy under Contract DEAT-03-81ER40002.

\section{Literature Cited}

1. Maki, Z., Nakazawa, M., Sakata, S. 1962. Prog. Theor. Phys. 28:870

2. Pontecorvo, B. 1967. Zh. Eksp. Theor. Fiz. 53:1717

3. Bilenky, S. M., Pontecorvo, B. 1978. Phys. Rep. 41:225

4. Frampton, P. H., Vogel, P. 1982. Phys. Rep. 82:339

5. Bullock, F. W., Devenish, R. C. E. 1983. Rep. Prog. Phys. 46: 1029

6. Cheng, T. P., Li, L. F. 1978. Phys. Rev. D $17: 2375$
7. Mohapatra, R. N., Senjanovic, G. 1980. Phys. Rev. Lett. 44:912

8. Wolfenstein, L. 1980. Nucl. Phys. B $175: 93$

9. Gell-Mann, M., Ramond, P., Slansky, R. 1970. In Supergravity, ed. P. van Nieuwenhuizen, p. 315. Amsterdam: North-Holland

10. Barbieri, R., Ellis, T., Gaillard, M. K. 1980. Phys. Lett. $90 \mathrm{~B}: 249$

11. Witten, E. 1980. Phys. Lett. $91 \mathrm{~B}: 81$

12. Turner, M. K. 1981. In Neutrino 81, ed. 
R. J. Cence, E. Ma, A. Roberts. Honolulu: Univ. Hawaii

13. Zeldovich, Ya. B., Sunyaev, R. A. 1980. Astron. Zh. Pisma 6:451

14. Tremaine, S., Gunn, J. E. 1979. Phys. Rev. Lett. 42:407

15. Schramm, D. N., Steigman, G. 1981. Astrophys. J. 243:1

16. Frenk, C. S., White, S. D. M., Davis, M. 1983. Astrophys. J. $271: 417$

17. Langacker; $P$., Leveille, J. P., Sheiman, J. 1983. Phys. Rev. D 27: 1228

18. Cabibbo, N., Maiani, L. 1982. Phys. Lett. $114 \mathrm{~B}: 115$

19. Davis, R., Jr., et al. 1983. Science Underground, AIP Conf. Proc., No. 96, p. 2. New York: AIP

20. Bahcall, J. N., et al. 1982. Rev. Mod. Phys. $54: 767$

21. Bahcall, J. N., Davis, R., Jr. 1982. In Essays in Nuclear Astrophysics, ed. C. A. Barnes, D. D. Clayton, D. N. Schramm. Cambridge: Cambridge Univ. Press

22. Wolfenstein, L. 1981, Phys. Lett. 107B: 77

23. Kayser, B., Goldhaber, A. S. 1983. Phys. Rev. D 28:2341

24. Barger, V., Langacker, P., Leveille, J. P., Pakvasa, S. 1980. Phys. Rev. Lett. 45:692

25. Kobzarev, I. Yu., Martemyanov, B. V., Okun, L. B., Shchepkin, M. G. 1981. Sov. J. Nucl. Phys. 32:823

26. DeRujula, A., Glashow, S. L. 1980. Phys. Rev. Lett. 45:942

27. Petcov, S. T. 1977. Sov. J. Nucl. Phys. $25: 340 ;(\mathrm{E}): 698$

28. Sato, E., Kobayashi, M. 1977. Prog. Theor. Phys. 58:1775

29. Pal, P. B., Wolfenstein, L. 1982. Phys. Rev. D 25:766

30. Reines, F., Sobel, M. W., Gurr, H. S. 1974. Phys. Rev. Lett. $32: 180$

31. Frank, J. S., et al. 1981. Phys. Rev. D 24:2001

32. Cowsik, R. 1980. In Neutrino Mass, ed. V. Barger, D. Cline, pp. 50-60. Univ. Wisc.

33. Bergsma, F., et al. 1983. Phys. Lett. 128B: 361

34. Toussaint, D., Wilczek, F. 1981. Nature 289:777

35. Shaevitz, M. 1983. In Int. Symp. on Lepton and Photon Interactions, Cornell Univ., Aug. 1983. Ithaca: Cornell Univ. Press

36. Cowan, C. L., Reines, F. 1957. Phys. Rev. 107:528

37. Wilkinson, D. H. 1982. Nucl. Phys. A $377: 474$

38. Vogel, P. 1984. Phys. Rev. D $29: 1918$

39. Reines, F., et al. 1980. Phys. Rev. Lett. $45: 1307$

40. Reines, F. 1983. Nucl. Phys. A 396:469
41. Vuilleumier, J.-L., et al. 1982. Phys. Lett. $114 \mathrm{~B}: 298$

42. Gabathuler, K., et al. 1984. Phys. Lett. 138B: 449

43. Kwon, H., et al. 1981. Phys. Rev. D $24: 1.097$

44. Schreckenbach, K., et al. 1981. Phys. Lett. 99B : 251

45. Vogel, P., et al. 1981. Phys. Rev. C 24: 1543

46. Boehm, F. 1984. AIP Conf. Proc. 112:1. New York : AIP

47. Von Feilitzsch, F., et al. 1982. Phys. Lett. 118B: 162

48. Shrock, R. E. 1980. Phys. Lett. 96B: 159 ; 1981. Phys. Rev. D 24:1232, 1275

49. Bergkvist, K. E. 1972. Nucl. Phys. B $39: 317,371$

50. Tretjakov, E. T., et al. 1976. Izv. Akad. Nauk. SSSR, Ser. Fiz. 40:20

51. Lyubimov, V. A., et al. 1980. Phys. Lett. 94B:266; 1981. Soc. Phys. JETP 54:616

52. Simpson, J. J. 1981. Phys. Rev. D 23:649

53. Boris, S., et al. 1983. In Proc. HEP 83, ed. J. Guy, C. Costain, pp. 386-89. Oxford: Rutherford Appleton Lab

54. Simpson, J. J. 1983. Proc. Int. Colloq. Matter Nonconservation, ed. E. Bellotti, S. Stipcich, p. 279. Frascati, Italy: Serv. Doc. Lab. Frascati

55. Kaplan, I. G., Smutny, V. N., Smelov, G. V. 1982. Phys. Lett. 112B: 417

56. Smith, L. G., Koets, E., Wapstra, A. H. 1981. Phys. Lett. 102B: 114

57. Simpson, J. J., Vogel, P. 1984. In Low Energy Tests of Conservation Laws, AIP Conf. Proc., 114:220. New York: AlP

58. De Rujula, A. 1981. Nucl. Phys. B 188: 414; 1982. Nucl Phys. A 379:619

59. Martin, P. C., Glauber, J. 1958. Phys. Rev. 109:1307

60. Ravn, H. L., et al. 1983. In Neutrino Mass and Gauge Structure of Weak Interactions, AIP Canf. Proc., No. 99, p. 1. New York: AIP

61. Andersen, J. V., et al. 1982. Phys. Lett. $113 \mathrm{~B}: 72$

62. Baisden, P. A., et al. 1983. Phys. Rev. C $28: 337$

63. Raghavan, R. S. 1983. Phys. Rev. Lett. $51: 975$

64. Beyer, G. J., et al. 1983. Nucl. Phys. A $408: 87$

65. Daum, M., et al. 1979. Phys. Rev. D $20: 2692$

66. Lu, D., et al. 1980. Phys. Rev. Lett. $45: 1066$

67. Abela, R., et al. 1983. SIN Newsletter $15: 26$

68. Anderhub, H. B., et al. 1982. Phys. Lett. 114B: 76

69. Clark, A. B., et al. 1974. Phys. Rev. D $9: 533$ 
70. Bacino, W., et al. 1979. Phys. Rev. Lett. 42:749

71. Blocker, C. A., et al. 1982. Phys. Lett. 109B : 119

72. Simpson, J. J. 1981. Phys. Rev. D 24: 2971

73. Schreckenbach, K., et al. 1983. Phys. Lett. 129B: 265

74. Simpson, J. J. 1981. Phys. Lett. 102B: 35

75. Bryman, D. A., et al. 1983. Phys. Rev. Lett. 50:1546

76. Dixit, M. S., et al. 1983. Phys. Rev. D 27:2216

77. Abela, P. 1981. Phys. Lett. 105B : 263

78. Hayano, R. S., et al. 1982. Phys. Rev. Lett. $49: 1305$

79. Rosen, S. P. 1982. Neutrino 81, 2:76. Honolulu: Univ. Hawaii

80. Doi, M., et al. 1983. Prog. Theor. Phys. $69: 602$

81. Bryman, D., Picciotto, C. 1978. Rev. Mod. Phys. 50:11; Zdesenko, Yu. G. 1980. Sov. J. Part. Nucl. 11:542

82. Kirsten, T., et al. 1983. Phys. Rev. Lett. $50: 474$

83. Hennecke, E, et al. 1975. Phys. Rev. C $11: 1378$

84. See for example Kirsten, T. 1982. In
Science Underground, AIP Conf. Proc. 96:396. New York: AIP; Boehm, F. 1982. AIP Conf. Proc. 93:321. New York: AIP; and Refs. 80 and 81

85. Haxton, W. C., Stephenson, G. J., Strottman, D. 1982. Phys. Rev. D 25:2360

86. Haxton, W. C., Stephenson, G. J., Strottman, D. 1981. Phys. Rev. Lett. 47:153

87. Moe, M., Lowenthal, D. 1980. Phys. Rev. C 22:2186

88. Cleveland, B., et al. 1975. Phys. Rev. Lett. 35:737

89. Moe, M. 1982. Neutrino 82, Proc. Int. Conf., ed. A. Frenkel, L. Jenik. Budapest, Hungary: Balatonfüred

90. Bellotti, E., et al. 1983. See Ref. 53

91. Avignone, F. T., et al. 1983. Phys. Rev. Lett. 50:721

92. Leccia, F. 1983. Univ. Bordeaux Preprint

93. Forster, A., et al. 1984. Phys. Lett. B 138B : 301

94. Forster, A., et al. 1984. The Time Projection Chamber, AIP Conf. Proc., $108: 56$. New York : AIP

95. Vogel, P. 1984. Phys. Rev. D. In press

96. Nieves, J. F. 1983. Phys. Rev. D 28:1664 
Annual Review of Nuclear and Particle Science
Volume 34, 1984

\section{CONTENTS}

Proton Decay Experiments, D. H. Perkins 1

NuCleosynthesis, James $W$. Truran 53

The Physics of Particle Accelerators, J. D. Lawson and $M$. Tigner $\quad 99$

Low-Energy Neutrino Physics and Neutrino Mass, F. Boehm and

$P$. Vogel

Nuclear Collisions at High Energies, S. Nagamiya, J. Randrup, and T. J. M. Symons

The Role of Rotational Degrees of Freedom in Heavy-Ion Collisions, L. G. Moretto and G. J. Wozniak

SUPERCONDUCTING MAGNET TECHNOLOGY FOR ACCELERATORS, R. Palmer and A. V. Tollestrup

High-Resolution Electronic Particle Detectors, G. Charpak and F. Sauli

HyPeron Beta DeCays, Jean-Marc Gaillard and Gilles Sauvage 351

Recent Progress in Understanding Trinucleon Properties,

J. L. Friar, B. F. Gibson, and G. L. Payne

Nuclear Reaction Techniques in Materials Analysis, G. Amsel and $W . A$. Lanford

Magnetic MoNOPOLES, John Preskill

Pion Interactions Within NuCLei, Mannque Rho

INDEXES

Cumulative Index of Contributing Authors, Volumes 24-34 